\title{
Thermodynamics of strongly interacting matter in a hybrid model
}

\author{
Abhijit Bhattacharyya, ${ }^{1, *}$ Sanjay K. Ghosh, ${ }^{2, \dagger}$ Soumitra Maity, ${ }^{2, *}$ Sibaji Raha, ${ }^{2, \S}$ Rajarshi Ray, ${ }^{2, \|}$ Kinkar Saha, ${ }^{1, \pi}$ \\ Subhasis Samanta, ${ }^{3, \#}$ and Sudipa Upadhaya ${ }^{4, * *}$ \\ ${ }^{1}$ Department of Physics, University of Calcutta, 92 A.P.C. Road, Kolkata 700009, India \\ ${ }^{2}$ Center for Astroparticle Physics \& Space Science, Block-EN, Sector-V, Salt Lake, Kolkata 700091, India \\ and Department of Physics, Bose Institute, 93/1, A.P.C. Road, Kolkata 700009, India \\ ${ }^{3}$ School of Physical Sciences, National Institute of Science Education and Research, HBNI, Jatni 752050, India \\ ${ }^{4}$ Variable Energy Cyclotron Centre, 1/AF Bidhannagar, Kolkata 700064, India
}

(Received 30 August 2017; revised manuscript received 27 December 2018; published 23 April 2019)

\begin{abstract}
The equation of state and fluctuations of conserved charges in a strongly interacting medium under equilibrium conditions form the baseline upon which are built various possible scenarios in relativistic heavy-ion collision experiments. Many of these quantities have been obtained in the lattice QCD framework with reliable continuum extrapolations. Recently the Polyakov-Nambu-Jona-Lasinio model has been reparametrized to some extent to reproduce quantitatively the lattice QCD equation of state at vanishing chemical potentials. The agreement was precise except at low temperatures, possibly due to inadequate representation of the hadronic degrees of freedom in the model. This disagreement was also observed for some of the fluctuations and correlations considered. Here we address this issue by introducing the effects of hadrons through the hadron resonance gas model. The total thermodynamic potential is now a weighted sum of the thermodynamic potential of the Polyakov-NambuJona-Lasinio model and that of the hadron resonance gas model. We find that the equation of state and the fluctuations and correlations obtained in this hybrid model agree satisfactorily with the lattice QCD data in the low temperature regime.
\end{abstract}

DOI: 10.1103/PhysRevC.99.045207

\section{INTRODUCTION}

The thermodynamic properties of strongly interacting matter under extreme conditions are being actively investigated both theoretically as well as experimentally. The lattice formulation of quantum chromodynamics $(\mathrm{QCD})$ on discretized space-time provides a first-principles approach in this direction [1]. In a system with light quarks a rapid crossover from color confined and chiral symmetry broken hadronic phase to a chirally restored and color deconfined partonic phase has been predicted [2-12]. In the physical case of two light quarks and a heavier strange quark, for lattice QCD (LQCD) simulations at zero net conserved charges, this crossover temperature

\footnotetext{
*abhattacharyyacu@gmail.com

†sanjay@jcbose.ac.in

¥soumitra.maity1984@gmail.com

§sibaji@jcbose.ac.in

"rajarshi@jcbose.ac.in

IIsaha.k.09@gmail.com

\#subhasis.samant@gmail.com

***sudipa.09@gmail.com
}

Published by the American Physical Society under the terms of the Creative Commons Attribution 4.0 International license. Further distribution of this work must maintain attribution to the author(s) and the published article's title, journal citation, and DOI. Funded by $S C O A P^{3}$. is expected to lie in the range $150<T_{c}<160 \mathrm{MeV}$ as reported by the Hot-QCD [13,14] and Wuppertal-Budapest [15] Collaborations. Though at a crossover there is no singularity, it is observed that near $T_{c}$ various thermodynamic quantities exhibit a rapid change [16-18].

At the same time, it is also important to properly explore the regions of the QCD phase diagram away from the temperature axis. It is expected that at some critical high baryon chemical potential and small temperatures the system may undergo a first-order phase transition from hadronic to partonic phase [19-21]. This first-order phase boundary would continue for some lower chemical potentials and higher temperature, eventually terminating at a critical endpoint (CEP) [22-25]. One of the major goals in experiments with heavy-ion collisions is to map this phase diagram of QCD and locate the CEP, if any.

A reliable way to understand the phase transition dynamics is through the study of correlations and fluctuations of conserved charges. At finite temperatures and chemical potentials fluctuations of conserved charges are sensitive indicators of the transition from hadronic matter to partonic matter. Moreover, the existence of the CEP can be signaled by the singularities in fluctuations. In the lattice QCD framework many of these susceptibilities have been obtained at vanishing chemical potentials. Unfortunately for nonzero baryon chemical potentials the lattice QCD framework faces certain technical difficulties. Recently various techniques have been developed to circumvent these difficulties to some extent [4,7,8,26-37]. 
A parallel approach with QCD inspired models is being developed alongside the lattice QCD approach to gain some insight into the various aspects of strongly interacting matter. Here we shall discuss one such model: the Polyakov loop enhanced Nambu-Jona-Lasinio (PNJL) model. Originally the PNJL model was introduced to enhance the Nambu-JonaLasinio (NJL) model [38-44] with gluon thermodynamics effectively through the Polyakov loop. In the absence of the gluon dynamics, the NJL model does not incorporate the mechanism oxf confinement adequately. Extending the NJL model to the PNJL model, by introducing a temporal background gluon-like field along with its self-interactions, restores some sense of confinement into the model [45-47]. The NJL as well as the PNJL models conserve all the global charges like the chirality, baryon number, electric charge, and strangeness, as in QCD. The multiquark interactions in this model are responsible for the dynamical generation of mass, leading to spontaneous breaking of chiral symmetry.

In the initial parametrizations of the PNJL model the NJL parameters were set from the masses of observed hadron masses and decay coefficients, while the Polyakov loop parameters were set from the pure gauge dynamics on the lattice. This already led to results qualitatively similar to the lattice QCD framework [47-54]. Over the years several studies were done to analyze the properties of this model as well as to improve the model step by step. For example, in order to stabilize the ground state of the $2+1$ flavor system, improvements were introduced in the PNJL model [55-57] following similar improvements in the NJL model [58-61]. A case study of the phase diagram in $\beta$ equilibrium using the PNJL model was reported in [62]. In a related work [63], the $\mathrm{SU}(3)$ color singlet ensemble of a quark-gluon gas was shown to exhibit a Z(3) symmetry, and within the stationary point approximation it becomes equivalent to the Polyakov loop ensemble. Significant effects on baryon-isospin correlations due to the mass difference between the two light quarks were reported in [64]. Similarly the effects of finite system sizes on various thermodynamic quantities, including fluctuations and correlations of conserved charges, have been reported in Refs. [65,66]. Also the first model study of the net charge fluctuations in terms of $D$ measure from the PNJL model [67] has been reported. In fact the validity of the fluctuation-dissipation theorem has also been discussed in the context of the PNJL model [68]. Viscous effects may play important role in the evolution of the hot and dense system. Their effects in terms of transport coefficients have been done in the NJL and PNJL models [69-76] and compared with hadron resonance gas studies [77-80]. The behavioral pattern of various observables extracted from the PNJL model may be found in $[62,81-92]$. The QCD phase structure has also been investigated for imaginary chemical potentials in the PNJL model framework [93-95]. Different interesting features of the Polyakov loop have led to the development of different formalisms of the PNJL model [63,96-98]. Effects of considering the gluon Polyakov loop have been discussed in Refs. [99-101]. Recently improvements of the Polyakov loop potential have been carried out by introducing the effects of back-reaction of the quarks $[102,103]$.
The qualitative agreement between the various observables computed in the PNJL model and those in lattice QCD has been quite satisfactory. This agreement seemed very convincing once the temperature dependent observables were plotted against $T / T_{c}$, where $T_{c}$ in the model was not equal to that obtained on the lattice. However lattice QCD data used for these studies were only reported at finite lattice spacings. Recently continuum extrapolations for a number of observables have been reported from lattice simulations [13,14,104,106-108]. Correspondingly the various thermodynamic properties of strongly interacting matter were investigated within the framework of the PNJL model by reparametrizing the Polyakov loop potential [109]. The focus was to ascertain a quantitative agreement of a variety of observables with the lattice data. The overall correspondence was satisfactory but not perfect. One of the regions of disagreement was in the low temperature region where hadronic degrees of freedom are dominant. This may be expected as these degrees of freedom are not adequately addressed in the PNJL model.

On the other hand, the hadron resonance gas (HRG) model [110] has been very successful in describing the hadron yields in central heavy-ion collisions from Alternating Gradient Synchrotron (AGS) up to Relativistic Heavy Ion Collider (RHIC) energies [111-123]. Along with that, the susceptibilities of conserved charges calculated in lattice QCD have been well reproduced by the HRG model [124-127] for temperatures up to $150 \mathrm{MeV}$. Also the region of large chemical potentials below the critical region can be studied using this model. Thus this model is quite suitable for describing the hadronic phase of strong interactions. The HRG model is based on the theorem of Dashen, Ma, and Bernstein [128] which shows that a dilute system of strongly interacting matter can be described by a gas of free resonances. Though the long range attractive part of the hadron interactions is taken care of by these resonances, the short range repulsion is also important for the description of strongly interacting matter. Near the critical/crossover region, HRG calculations tend towards Hagedorn divergence, which may be due to the absence of repulsive interaction. This repulsive part is incorporated through the excluded volume effects in the HRG and is commonly known as the EVHRG model [126,129-138]. EVHRG equations of state have also been used for the hydrodynamic models of nucleus-nucleus collision [139-141]. Recently fluctuations of conserved charges, using the HRG model, were studied in Ref. [142] including the experimental acceptances of pseudorapidity and transverse momentum. In Ref. [137], higher moments of net-proton multiplicity were studied using the EVHRG model and compared with STAR experiment data. A similar study was done by some of us for net proton, net charge, and net kaon using the HRG and EVHRG models [127]. The effects of finite volumes on the hadron gas were discussed in [143] and effects of magnetic fields were presented in [144]. In fact, as the higher moments are expected to be more sensitive to the phase transition, any deviation of experimental observation from the model results may be taken as an indication of new phenomena. Investigation in this direction was done with variation of collision centrality as well as collision energy in [145]. 
Unfortunately, there is no single model which describes both the low temperature and high temperature domains accurately. However, a possible mechanism to address this issue has been suggested in [146]. The idea is to make an interface between one model appropriate for the hadronic phase and another model appropriate for the partonic phase. For the hadronic sector the authors used the HRG model, and for the partonic sector they used the PQCD results, and they used a switching function at the interface. In a followup work [147] they discussed the various versions of HRG models along with the PQCD data and obtained quite a number of thermodynamic variables including some susceptibilities in the baryon sector. They found the equation of state to be reproduced very well vis-à-vis the lattice QCD data, but the values of some of the susceptibilities deviate slightly from the lattice QCD data. However the baryonic susceptibilities obtained in STAR data are well reproduced with the EVHRG model for the hadronic sector [148].

In a similar study an interacting quark (IQ) model, which is a modified PNJL model, was used for the partonic phase in $[149,150]$ and the HRG model was used for the hadronic phase. Here the multiquark interactions in the NJL sector were neglected and the quark masses were taken to be the nominal Lagrangian masses. In these studies the equation of state and susceptibilities of various conserved charges were computed, and they reproduced the lattice QCD data quite well.

The above approaches seem to go against the usual notion of two completely different phases corresponding to hadrons and partons. But the hadrons and partons are states of the same underlying theory of strong interactions. So in principle nothing precludes these states from appearing simultaneously in a given thermodynamic condition. In the light of lattice QCD results indicating a crossover of hadron domination to parton domination, such a mixture of states is quite possible in the crossover region. Therefore it is worth exploring models with coexisting hadronic and partonic degrees of freedom. The possible existence of such ensembles where both bound and unbound states can coexist has already been discussed in general quantum statistical systems, and for hadron-parton transitions in particular [151]. It has been argued that though a given thermodynamic condition may prefer certain degrees of freedom over the others, there is always a possibility that all the degrees of freedom coexist in another thermodynamic domain. In other words there may be thermodynamic regions where either the hadronic or the partonic degrees of freedom are preferred, but there may still be conditions where both may coexist. The coexistence region need not always be thought of as hadronic phase coexisting with partonic phase, as would be the case at a first-order phase transitions. Rather the coexistence may be at the level of a simple mixture of partons and hadrons. Such a picture would be most suitable for a crossover from a region of hadron dominance to parton dominance. The switching functions in Refs. [146-150] may thus be related to the clustering probability in Ref. [151].

In the present study we shall explore this approach utilizing the PNJL model and the HRG model. Before going on to the details let us clarify some of our assumptions and some of the differences with the earlier studies. Here we shall consider the simplest noninteracting form of the HRG model to describe the low temperature phase. We expect the effects of the short range repulsive forces incorporated in the excluded volume HRG model to be small for $T<150$ Mev [127]. As we shall see, the noninteracting HRG model easily describes most of the physics in the low temperature phase.

Secondly, in both sets of the earlier studies the switching function is considered to have both temperature and chemical potential dependence. This should be true in general. However, for simplicity we have chosen it to be temperature dependent, as in the present work we shall only concentrate on observables at zero chemical potential. It is to be noted that the switching function itself does not affect any properties of either the PNJL model or the HRG model. This means that the chiral and/or deconfinement transition as decided from the characteristics of the chiral condensate and the Polyakov loop respectively are insensitive to the switching function, thus keeping the phase boundary dependent only on the PNJL model. This would in turn mean that we would be constrained to keep the changeover temperature in the switching function close to the crossover temperature in the PNJL model, as will be the case discussed later.

Lastly, all the earlier studies have considered derivatives of the switching function for computing further observables using the thermodynamic relations. For example, if the switching function is used as an interpolating function of hadronic and partonic pressure, the entropy will be obtained from the temperature derivative, which also acts on the switching function. The switching function is required mainly for two reasons. First, we want to consider the contribution of hadrons at low temperatures, which is absent in the PNJL model. This is done by adding the HRG model. Second, we want to suppress the contribution of hadrons at high temperatures where partonic phase dominates. Note that the partonic contribution is already suppressed by the Polyakov loop for low temperatures, and the switching function in that region is superfluous. Nonetheless the switching function is supposed to give the proportion of the hadronic and partonic contributions. The usual approach is to choose an observable like pressure (in our case) or entropy (as in $[149,150]$ ) and impose the switching function on that. Subsequently the other thermodynamic quantities are obtained by usual thermodynamic relations. This seems to necessitate the use of the derivatives of the switching function for thermodynamic consistency. We instead take an alternative approach that all the thermodynamic quantities be first obtained both in hadronic and partonic models at a given temperature and chemical potential. Thereafter we choose a unique proportion for the hadronic and partonic contributions for all the extracted observables and try to reproduce LQCD data. However, we have no reason to exclude the former approach and therefore we examine both these situations side by side. As we discuss below, with proper parametrizations the numerical results do not depend much on whether or not the derivatives are included. Similar derivatives of the switching functions were considered in the IQ-HRG approach [150], where the derivative terms were used as parameters to fit the lattice 
TABLE I. Parameters in the NJL model.

\begin{tabular}{lccccccc}
\hline \hline Interaction & $m_{u}(\mathrm{MeV})$ & $m_{s}(\mathrm{MeV})$ & $\Lambda(\mathrm{MeV})$ & $g_{s} \Lambda^{2}$ & $g_{D} \Lambda^{5}$ & $g_{1} \times 10^{-21}\left(\mathrm{MeV}^{-8}\right)$ & $g_{2} \times 10^{-22}\left(\mathrm{MeV}^{-8}\right)$ \\
\hline 6-quark & 5.5 & 134.758 & 631.357 & 3.664 & 74.636 & 0.0 & 0.0 \\
8-quark & 5.5 & 183.468 & 637.720 & 2.914 & 75.968 & 2.193 & -5.890 \\
\hline \hline
\end{tabular}

QCD data for entropy and susceptibilities. Since we shall not consider the chemical potential dependence of the switching function, these derivative effects in the susceptibilities in our study are zero. Yet we shall see that the results seem to satisfactorily reproduce lattice QCD data in the hadronic phase.

We have organized the paper as follows. In the next two sections, II and III, we shall briefly discuss the PNJL model and the HRG model respectively. In Sec. IV we discuss how to couple the PNJL and HRG models, utilizing some of the thermodynamic quantities relating to the equation of state. Thereafter in Sec. V we discuss the predicted behavior of the fluctuations and correlations of conserved charges from the PNJL-HRG model. Finally in Sec. VI we summarize and conclude.

\section{PNJL MODEL}

In this section we briefly discuss the PNJL model used in this study. A detailed discussion may be found in Ref. [109]. The PNJL model was initialized with a Polyakov loop effective potential being added to the NJL model [45-47]. While the chiral properties are taken care of by the NJL part, the Polyakov loop explains the deconfinement physics. Extensive studies have been carried out using PNJL model with 2 and $2+1$ flavors [47-49,51,55,152-156]. A more consistent approach with regularized thermal contribution was discussed in [157]. The main result is the correct asymptotic behavior of the quark masses approaching the current masses. Here we consider $2+1$ flavor PNJL model taking up to six and eight quark interaction terms as in $[55,152]$. The thermodynamic potential in this case reads as

$$
\begin{aligned}
\Omega\left(\Phi, \bar{\Phi}, \sigma_{f}, T, \mu\right)= & 2 g_{S} \sum_{f=u, d, s} \sigma_{f}^{2}-\frac{g_{D}}{2} \sigma_{u} \sigma_{d} \sigma_{s}+3 \frac{g_{1}}{2}\left(\sum_{f} \sigma_{f}^{2}\right)^{2}+3 g_{2} \sum_{f} \sigma_{f}^{4}-6 \sum_{f} \int_{0}^{\infty} \frac{d^{3} p}{(2 \pi)^{3}} E_{f} \Theta(\Lambda-|\vec{p}|) \\
& -2 T \sum_{f} \int_{0}^{\infty} \frac{d^{3} p}{(2 \pi)^{3}} \ln \left[1+3\left(\Phi+\Phi e^{-\left(E_{f}-\mu_{f}\right) / T}\right) e^{-\left(E_{f}-\mu_{f}\right) / T}+e^{-3\left(E_{f}-\mu_{f}\right) / T}\right] \\
& -2 T \sum_{f} \int_{0}^{\infty} \frac{d^{3} p}{(2 \pi)^{3}} \ln \left[1+3\left(\bar{\Phi}+\Phi e^{-\left(E_{f}+\mu_{f}\right) / T}\right) e^{-\left(E_{f}+\mu_{f}\right) / T}+e^{-3\left(E_{f}+\mu_{f}\right) / T}\right]+\mathcal{U}^{\prime}(\Phi, \bar{\Phi}, T)
\end{aligned}
$$

The fields $\sigma_{f}=\left\langle\bar{\psi}_{f} \psi_{f}\right\rangle$ correspond to the two light flavor $(f=u, d)$ condensates and the strange $(f=s)$ quark condensate respectively. The model has a four-quark coupling term with coefficient $g_{S}$, a six-quark coupling term breaking the axial U(1) symmetry explicitly with a coefficient $g_{D}$, and eight-quark coupling terms with coefficients $g_{1}$ and $g_{2}$ necessary to sustain a stable minima in the NJL Lagrangian. The corresponding quasiparticle energy for a given flavor $f$ is $E_{f}=\sqrt{p^{2}+M_{f}^{2}}$, with the dynamically generated constituent quark masses given by

$$
\begin{aligned}
M_{f}= & m_{f}-2 g_{S} \sigma_{f}+\frac{g_{D}}{2} \sigma_{f+1} \sigma_{f+2} \\
& -2 g_{1} \sigma_{f}\left(\sigma_{u}^{2}+\sigma_{d}^{2}+\sigma_{s}^{2}\right)-4 g_{2} \sigma_{f}^{3}
\end{aligned}
$$

In the above, if $\sigma_{f}=\sigma_{u}$, then $\sigma_{f+1}=\sigma_{d}$ and $\sigma_{f+2}=\sigma_{s}$, and so on in a clockwise manner. The finite range integral gives the zero point energy. The different parameters as obtained from [55] are given in Table I.

The finite temperature and chemical potential contributions of the constituent quarks are given by the next two terms. Note that these are basically coming from the fermion determinant in the NJL model modified due to the presence of the fields corresponding to the traces of Polyakov loop and its conjugate given by $\Phi=\frac{T r_{c} L}{N_{c}}$ and $\bar{\Phi}=\frac{T r_{c} L^{\dagger}}{N_{c}}$ respectively. Here $L(\vec{x})=$ $\mathcal{P} \exp \left[i \int_{0}^{1 / T} d \tau A_{4}(\vec{x}, \tau)\right]$ is the Polyakov loop, and $A_{4}$ is the temporal component of background gluon field.

The effective potential that describes the self-interaction of the $\Phi$ and $\bar{\Phi}$ fields is given by $\mathcal{U}^{\prime}$. Various forms of the potential exist in the literature (see, e.g., [50,51,83,158,159]). We shall use the form prescribed in [51], which reads as

$$
\frac{\mathcal{U}^{\prime}(\Phi, \bar{\Phi}, T)}{T^{4}}=\frac{\mathcal{U}(\Phi, \bar{\Phi}, T)}{T^{4}}-\kappa \ln [J(\Phi, \bar{\Phi})]
$$

Here $\mathcal{U}(\Phi, \bar{\Phi}, T)$ is a Landau-Ginzburg type potential commensurate with the global Z(3) symmetry of the Polyakov loop [47]. $J(\Phi, \bar{\Phi})$ is the Jacobian of transformation from the Polyakov loop to its traces, and $\kappa$ is a dimensionless parameter which is determined phenomenologically. The effective potential is chosen to be of the form

$$
\frac{\mathcal{U}(\Phi, \bar{\Phi}, T)}{T^{4}}=-\frac{b_{2}(T)}{2} \bar{\Phi} \Phi-\frac{b_{3}}{6}\left(\Phi^{3}+\bar{\Phi}^{3}\right)+\frac{b_{4}}{4}(\bar{\Phi} \Phi)^{2}
$$


TABLE II. Parameters for the Polyakov loop potential.

\begin{tabular}{lcccccc}
\hline \hline Interaction & $T_{0}(\mathrm{MeV})$ & $a_{0}$ & $a_{1}$ & $a_{2}$ & $b_{3}$ & $b_{4}$ \\
\hline 6-quark & 175 & 6.75 & -9.0 & 0.25 & 0.805 & 7.555 \\
8-quark & 175 & 6.75 & -9.8 & 0.26 & 0.805 & 7.555 \\
\hline \hline
\end{tabular}

The coefficient $b_{2}(T)$ is chosen to have a temperature dependence of the form

$$
b_{2}(T)=a_{0}+a_{1} \exp \left(-a_{2} \frac{T}{T_{0}}\right) \frac{T_{0}}{T},
$$

and $b_{3}$ and $b_{4}$ are chosen to be constants. The set of parameters is given in Table II.

Using realistic quark masses, the deconfinement temperature obtained in lattice QCD is found to be much higher than the chiral transition temperature $[9,11]$. However, the deconfinement temperature as measured from the peak of the entropy of a static quark is found to be consistent with the chiral transition temperature [160]. In our model framework we consider the temperature derivatives of the mean fields and locate their peaks to obtain the transition temperature. The temperature derivative of the Polyakov loop is closely related to the definition of temperature derivative of the static quark free energy that gives its entropy as defined in [160]. The corresponding $T_{c}$ was obtained from the average of the two peak positions. The resulting values of $T_{c}$ are listed in Table III.

With this parametrization we have been able to achieve both a crossover temperature of $T_{c} \sim 160 \mathrm{MeV}$ as well as quantitative agreement of temperature variation of pressure and various other observables with the lattice QCD continuum estimation [109]. However, the quantitative agreement, though close, was not precise enough. Various observables showed disagreement in different ranges of temperatures. The most common discrepancies were found in the low temperature region where the hadronic degrees of freedom dominate. We shall thus make an attempt to remove this lacunae by coupling the PNJL model with the HRG model.

\section{HADRON RESONANCE GAS MODEL}

We now discuss the HRG model briefly. Detailed discussions may be found in Refs. [110-115,117,118,120,126,129-133]. The grand canonical partition function of a hadron resonance gas $[110,126]$ can be written as

$$
\ln Z^{i d}=\sum_{i} \ln Z_{i}^{i d}
$$

where the sum is over all the hadrons. id refers to an ideal gas of hadronic resonances. The partition function for the $i$ th resonance is

$$
\ln Z_{i}^{i d}= \pm \frac{V g_{i}}{2 \pi^{2}} \int_{0}^{\infty} p^{2} d p \ln \left\{1 \pm \exp \left[-\left(E_{i}-\mu_{i}\right) / T\right]\right\},
$$

where $V$ is the volume of the system, $g_{i}$ is the degeneracy factor, $T$ is the temperature, $E_{i}=\sqrt{p^{2}+m_{i}^{2}}$ is the single-particle energy, $m_{i}$ is the mass, and $\mu_{i}=B_{i} \mu_{B}+S_{i} \mu_{S}+Q_{i} \mu_{Q}$ is the chemical potential. $B_{i}, S_{i}, Q_{i}$ are respectively the baryon number, strangeness, and charge of the particle, $\mu$ 's being the corresponding chemical potentials. The $(+)$ and $(-)$ signs correspond to fermions and bosons respectively. From the partition function one can calculate various thermodynamic observables of the system created in heavy-ion collisions. In this work we shall incorporate all the hadrons listed by the Particle Data Group [161] up to a mass of $3 \mathrm{GeV}$.

\section{COUPLING THE HADRONIC AND PARTONIC SECTORS}

Let us now discuss the framework in which the hadronic matter described using the HRG model can be smoothly switched to partonic matter modeled through the PNJL one. The basic procedure is similar to the ones reported in [146-150]. The pressure of the system as taken to be a sum of partial pressures of the hadronic and partonic matter, weighted with a switching function, as

$$
P(T)=S(T) P_{P}(T)+[1-S(T)] P_{H}(T),
$$

where $P_{P}(T)$ and $P_{H}(T)$ are the pressures of partonic and hadronic sectors respectively and $S(T)$ is the switching function. For the partonic pressure we shall make a comparative study between PNJL models with six and eight quark type interactions respectively.

Ideally $S(T)$ should be 0 in the hadronic phase and 1 in the partonic phase. However since there is no singular phase boundary separating the two phases, we consider the switching function to interpolate smoothly from 0 at low temperatures to 1 at high temperatures. We have assumed the switching function to be independent of chemical potential at zero chemical potential. The functional form of the switching function is given as

$$
S(T)=\frac{1}{1+\exp \left[-\frac{T-T_{S}}{\Delta T_{S}(T)}\right.} .
$$

TABLE III. Location of crossover temperature.

\begin{tabular}{lccc}
\hline \hline Interaction & Peak position of $d \Phi / d T(\mathrm{MeV})$ & Peak position of $d \sigma / d T(\mathrm{MeV})$ & $T_{c}(\mathrm{MeV})$ \\
\hline 6-quark & 142 & 191 & 166.5 \\
8-quark & 158 & 167 & 162.5 \\
\hline \hline
\end{tabular}


Here $T_{S}$ and $\Delta T_{S}(T)$ are parameters whose values should be closely related to the crossover temperature and width of the crossover region respectively. Note that the form of the switching function required is essentially that of any kind of a sigmoid function. The simplest that can be considered are the hyperbolic tangent and logistic functions. We have chosen the latter as we found it is naturally bounded between 0 and 1 , unlike the hyperbolic tangent which is bounded between -1 and 1 . However, we also tried the hyperbolic tangent function and got similar outcomes. Note that there are two temperature scales: one being the central value $T_{S}$ of the switching and the other being the spread $\Delta T_{S} . T_{S}$ is related to the crossover temperature and $\Delta T_{S}(T)$ to the spread of the switching function on the temperature axis from its central value. Given that the crossover temperature is not sharply defined, we have let $T_{S}$ be fixed at $160 \mathrm{MeV}$. Thereafter we try to find the best possible value of $\Delta T_{S}(T)$ so as to obtain the closest quantitative agreement of pressure as obtained in lattice QCD.

The procedure for obtaining the various thermodynamic observables is as follows. We first obtain the pressure in the HRG model as well as the PNJL model from the respective thermodynamic potentials. Thereafter we obtain the hybrid pressure according to Eq. (8). Once we have the pressure, the other thermodynamic quantities like entropy, and specific heat, are obtained from the temperature derivatives of the hybrid pressure. In all the previous studies the temperature and chemical potential derivatives of the switching function in the hybrid pressure were included as well. We, however, consider the switching function to be defined globally for all the observables considered, so that the effective function should be independent of the observable being measured. But we shall still make a comparative study by both including and excluding the temperature derivative terms for some of the thermodynamic variables.

The entropy density is obtained from the first derivative of pressure with respect to temperature and is given by

$$
\begin{aligned}
s(T)= & \frac{\partial P}{\partial T}=\left[S(T) s_{P}(T)+(1-S(T)) s_{H}(T)\right] \\
& +\left(P_{P}(T)-P_{H}(T)\right) \frac{\partial S(T)}{\partial T}
\end{aligned}
$$

Here $s_{P}(T)$ and $s_{H}(T)$ are the entropy densities for the PNJL model and HRG model respectively. Note that the term in the square brackets denotes the hybrid entropy due to the switching function, while the other term denotes the effects of the derivatives of the switching function itself. The case would be similar for any other thermodynamic quantity where temperature derivative is involved. For example the energy density is given by

$$
\begin{aligned}
\epsilon(T)= & T s(T)-P(T)=\left[S(T) \epsilon_{P}(T)+(1-S(T)) \epsilon_{H}(T)\right] \\
& +T\left(P_{P}(T)-P_{H}(T)\right) \frac{\partial S(T)}{\partial T} .
\end{aligned}
$$

Here again the term within the square brackets would give the hybrid energy density and the other term comes from the

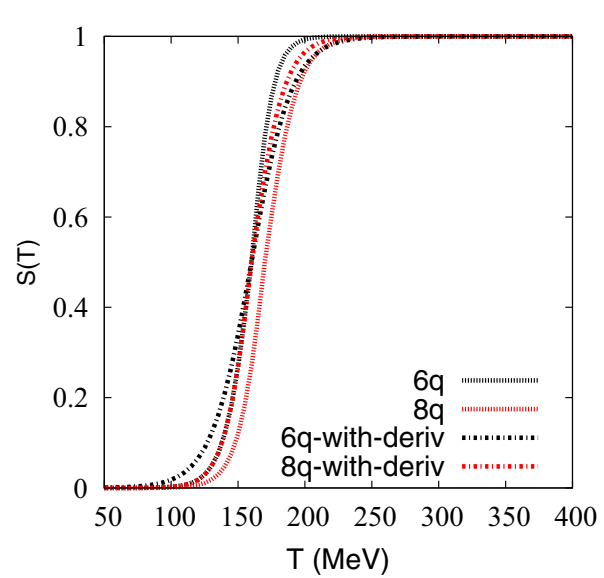

FIG. 1. Switching function as a function of temperature.

derivative of $S(T)$. The derivative of $S(T)$ is given as

$$
\frac{\partial S(T)}{\partial T}=\frac{1}{\Delta T_{S}}\left[S(T)-S(T)^{2}\right]
$$

The derivatives of $S(T)$ contribute more terms for higher order temperature derivatives. For the specific heat at constant volume we get the expression as

$$
\begin{aligned}
C_{V}(T)= & \left(\frac{\partial \epsilon}{\partial T}\right)_{V}=\left[S(T) C_{V P}(T)+(1-S(T)) C_{V H}(T)\right] \\
& +2 T\left(s_{P}(T)-s_{H}(T)\right) \frac{\partial S(T)}{\partial T} \\
& +T\left(P_{P}(T)-P_{H}(T)\right) \frac{\partial^{2} S(T)}{\partial T^{2}}
\end{aligned}
$$

Here the secondorder derivative term of $S(T)$ is given as

$$
\frac{\partial^{2} S(T)}{\partial T^{2}}=\frac{1}{\left(\Delta T_{S}\right)^{2}}\left[S(T)-3 S(T)^{2}+2 S(T)^{3}\right] .
$$

We now show the temperature variations of some of the thermodynamic quantities. We shall compare the results in the PNJL model with six and eight quark interactions. We shall also compare results including and excluding the temperature derivatives of the switching function. As mentioned earlier the only parameter adjusted in the switching function is $\Delta T_{S}(T)$. We have also allowed it to take two different values on the two sides of $T_{S}$ when the derivatives of $S(T)$ are excluded, as that seemed to improve the agreement of the model results with LQCD data (with the derivatives included this cannot be done as the derivatives would diverge). Here the values of $\Delta T_{S}(T)$ are not uniquely determined by any standard fitting procedure. However, for the purpose of this paper it suffices to choose the values that best describe most of the thermodynamic quantities in the hybrid systems by trial and error. These values of $\Delta T_{S}(T)$ are given in Table (IV). Results are not much affected by shifting these values by $10 \%$. The form of the switching function is shown in Fig. 1.

In Fig. 2 we show scaled pressure as a function of temperature for various models and compare them to the lattice QCD data $[14,15]$. While the HRG model agrees with lattice QCD data below $T=200 \mathrm{MeV}$, the PNJL model agrees with 

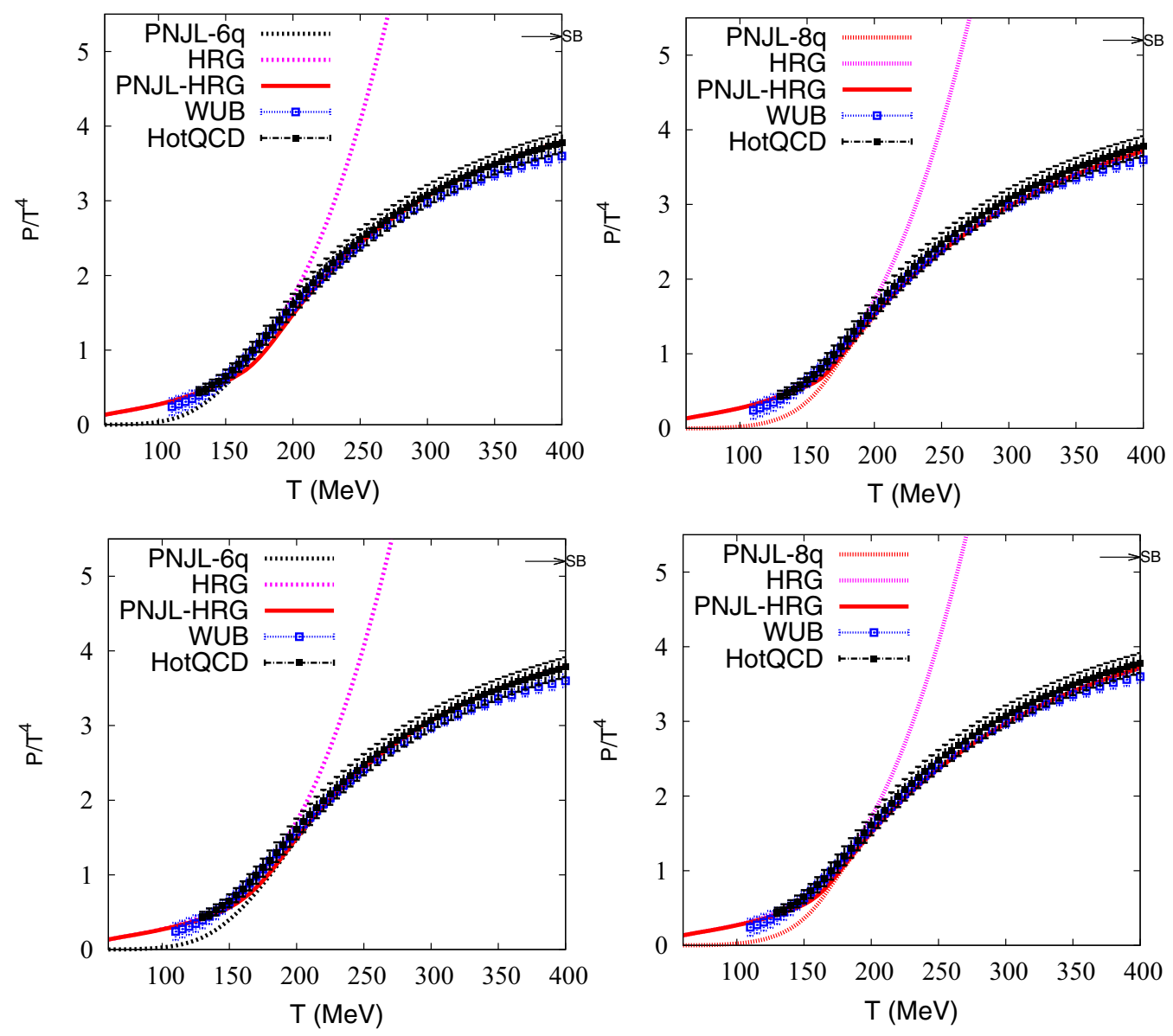

FIG. 2. Scaled pressure as a function of temperature. The upper panels are drawn without derivatives of switching function and lower panels include those derivatives. The left panel is for the PNJL model with six quark interactions and the right panel for the PNJL model with eight quark interactions. The continuum extrapolated lattice QCD data are taken from Ref. [14] (HotQCD) and Ref. [15] (WUB).

lattice QCD data above $T=150 \mathrm{MeV}$. On the other hand the combined PNJL-HRG model now agrees with the lattice QCD data for the full temperature range. The differences in the results due to the type of PNJL model chosen are quite insignificant. However, some of those effects are absorbed in the values of $\Delta T_{S}$ as given in Table IV. We note here that the pressure itself only depends on $S(T)$ and not on any of its derivatives. However the other thermodynamic variables are obtained from the derivatives of the hybrid pressure. As mentioned earlier, we are choosing $\Delta T_{S}$ such that the bulk thermodynamic quantities obtained in lattice QCD framework are well reproduced in the PNJL-HRG model. Thus the values of pressure would depend on which form of $S(T)$ we are choosing. This is why we also show the plot for pressure for those values of $\Delta T_{S}$ that are chosen when derivatives of $S(T)$ are included in computing the rest of the thermodynamic variables.

The two other quantities that we considered for critically assessing the quantitative agreement with lattice QCD data are the trace of the energy-momentum tensor and the squared speed of sound. The trace of the energy-momentum tensor is defined as $\Theta_{\mu \mu}=(\epsilon-3 P)$. It is expected to be zero in a conformal theory. However, the introduction of any scale like the crossover or transition temperatures in the theory due to quantum interactions would break conformality and the trace will be nonzero. This is expected to be a very sensitive measure, inherent to the theory itself. Therefore a satisfactory agreement of the results obtained from lattice

TABLE IV. Values of $\Delta T_{S}$ used.

\begin{tabular}{|c|c|c|c|}
\hline \multirow[t]{2}{*}{ PNJL interaction } & \multirow{2}{*}{$\begin{array}{c}\Delta T_{S}(\mathrm{MeV}) \\
\frac{\text { including derivatives of } S(T)}{\text { For all values of } \mathrm{T}}\end{array}$} & \multicolumn{2}{|c|}{$\begin{array}{c}\Delta T_{S}(\mathrm{MeV}) \\
\text { excluding derivatives of } S(T)\end{array}$} \\
\hline & & $T<T_{S}$ & $T>T_{S}$ \\
\hline 6-quark & 15 & 10 & 8 \\
\hline 8-quark & 15 & 10 & 18 \\
\hline
\end{tabular}



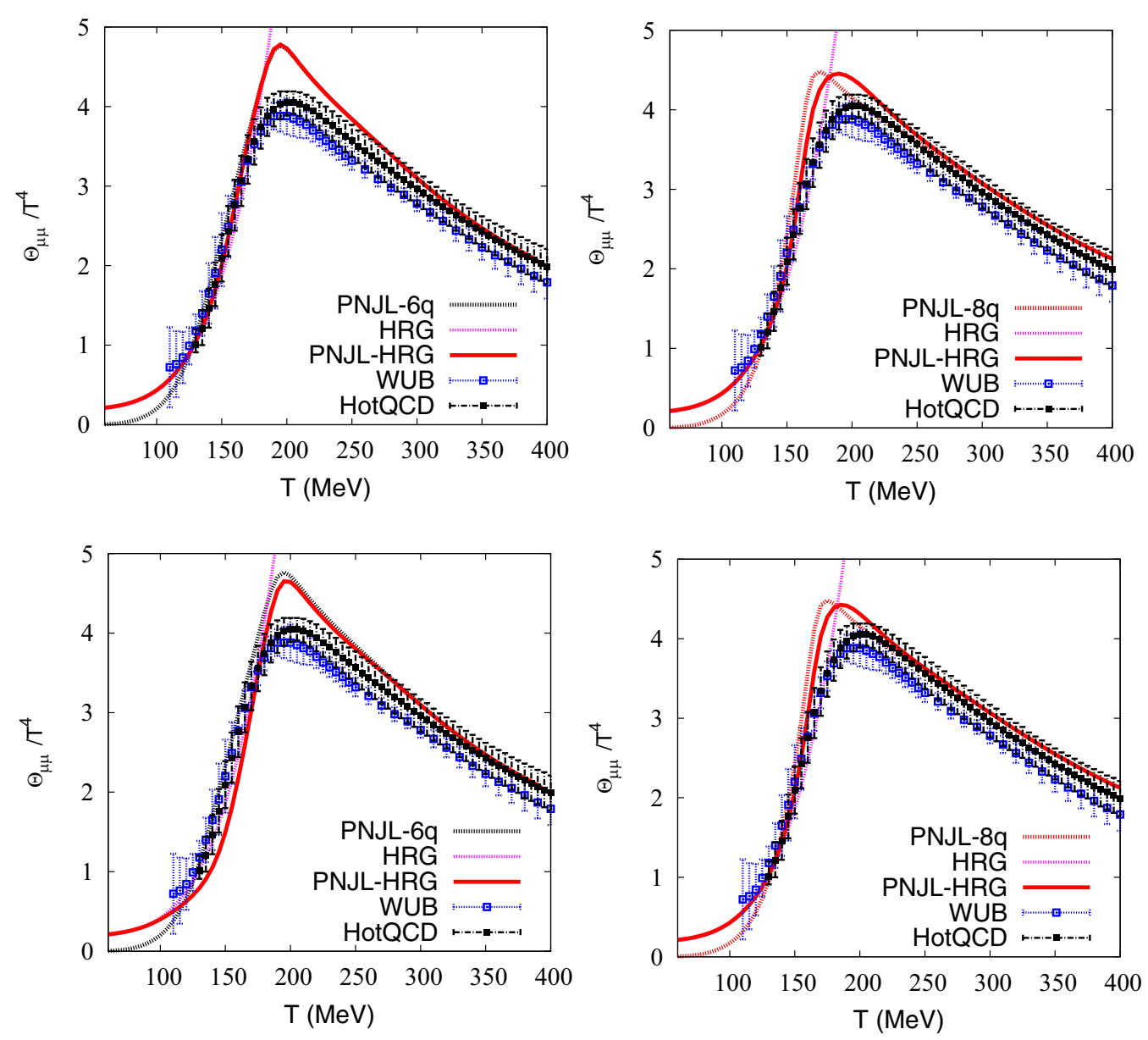

FIG. 3. The scaled traces of the energy-momentum tensor are plotted as a function of temperature. The upper panels are drawn without derivatives of the switching function and lower panels include those derivatives. The left panel is for the PNJL model with six quark interactions and the right panel is for the PNJL model with eight quark interactions. The continuum extrapolated lattice QCD data are taken from Ref. [14] (HotQCD) and Ref. [15] (WUB).

QCD with any other model is imperative for validation of the model as a simpler version of the theory.

In Fig. 3 we show scaled $\Theta_{\mu \mu}$ as a function of temperature for various models and compare them to the lattice QCD data $[14,15]$. Here the HRG model agrees with lattice QCD data up to about $T=170 \mathrm{MeV}$ and rises much faster for higher temperatures. The PNJL model agrees with lattice QCD data above $T=150 \mathrm{MeV}$, except near the peak at around $T=200$ $\mathrm{MeV}$. Apart from this peak region, the combined PNJL-HRG model now agrees with the lattice QCD data for the full temperature range. The differences in the results due to the type of PNJL model chosen are again quite small. However, near the peak region the eight-quark version of the PNJL model better describes the lattice QCD data and so does the PNJL-HRG model. The deviation from the lattice QCD data near the peak region would need further improvement of the PNJL model as the switching function favors it over the HRG model in this temperature range. Since our motivation here is to improve the situation for the temperature region dominated by hadrons, we do not intend to address this discrepancy here.

We now discuss the behavior of the speed of sound as a function of temperature. The squared speed of sound is given by

$$
c_{s}^{2}=\frac{\partial P}{\partial \epsilon}=\frac{s}{C_{V}} .
$$

The variation of the squared speed of sound with temperature is shown in Fig. 4 for various models and compared with the lattice QCD data [14,15]. As seen from Eq. (14), the speed of sound on one hand contains the information of the equation of state and on the other hand contains the information of the ratio of entropy to specific heat. Thus the characteristics of the speed of sound depend strongly on the phase of strongly interacting matter. One expects that at very low temperatures the speed of sound would be small, as the pressure of the hadronic system with masses much larger than the system is negligible. With increase in temperature the speed of sound will increase. However, with increasing temperature the hadron resonances with higher and higher masses would be excited and the speed of sound may not reach the SB limit. In fact it may even start decreasing with temperature [126]. Using the HRG model all these features are obtained as shown in Fig. 4. After the crossover, the degrees of freedom are supposed to change from hadronic to partonic ones and therefore the speed of sound may again 

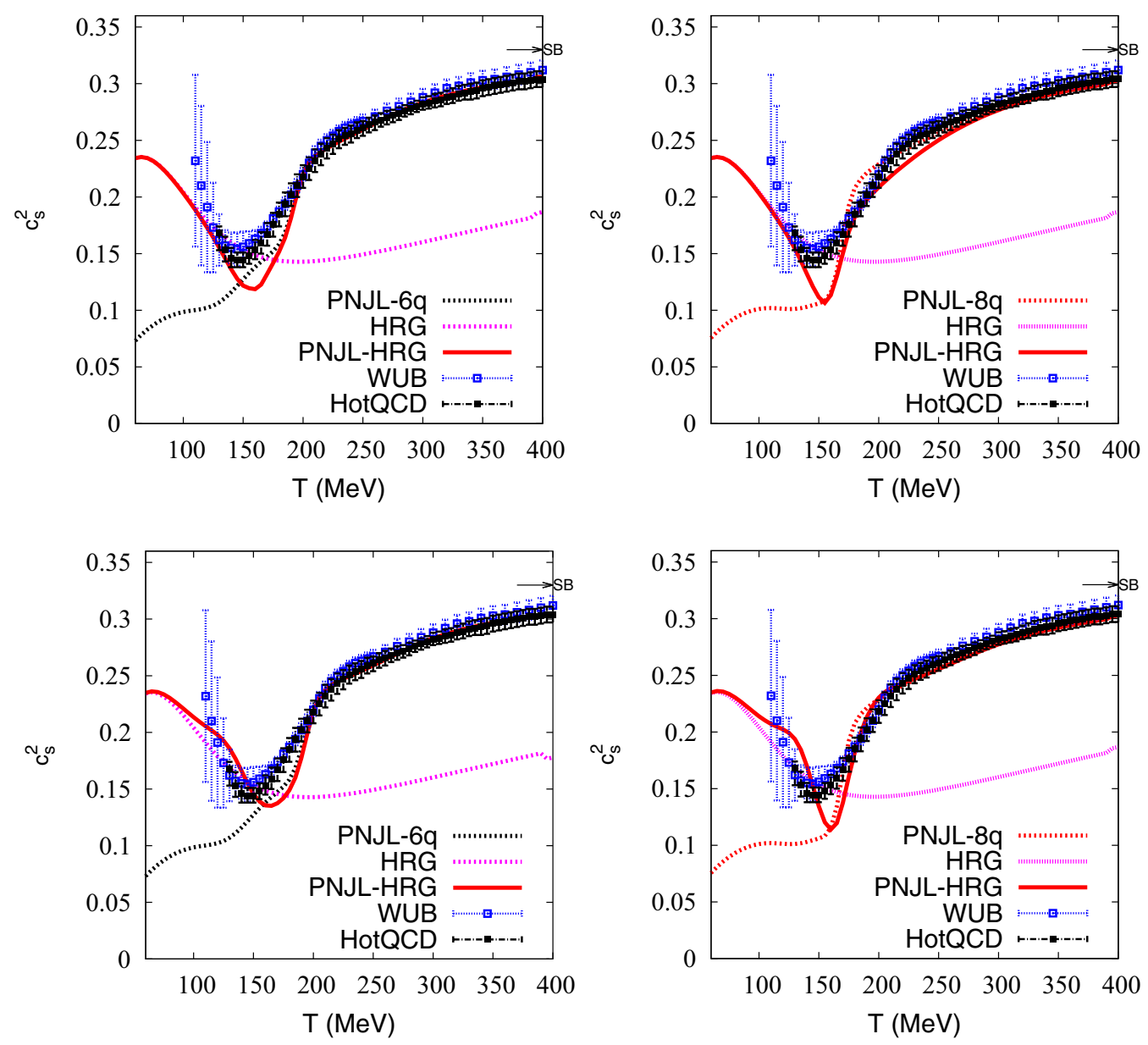

FIG. 4. The squared speed of sound is plotted as a function of temperature. The upper panels are drawn without derivatives of the switching function and the lower panels include those derivatives. The left panel is for the PNJL model with six quark interactions and the right panel is for PNJL model with eight quark interactions. The continuum extrapolated lattice QCD data are taken from Ref. [14] (HotQCD) and Ref. [15] (WUB).

increase. The minimum of the speed of sound known as the softest point may be a crucial indicator of the transition to be observed in heavy-ion collisions [162]. Such a minimum in the temperature variation of speed of sound is visible in the lattice QCD data as shown in Fig. 4, but is clearly absent in the PNJL model results. It is interesting to see that even the HRG model has a soft point of the same order of magnitude but at a higher temperature compared to the lattice QCD data. While the HRG model agrees with lattice QCD data up to about $T=150 \mathrm{MeV}$, the PNJL model results are consistent with the lattice data above $T_{c}$. Thus the combined PNJL-HRG model shows reasonable agreement with the lattice QCD data in the full range of temperatures.

From the above discussions we may conclude that a hybrid model like PNJL-HRG satisfactorily describes the equation of state of strongly interacting matter in a wide range of temperatures. We would now like to investigate whether the same holds true for the various fluctuations and correlations at vanishing chemical potentials.

\section{FLUCTUATIONS AND CORRELATIONS OF CONSERVED CHARGES}

In strong interactions the net numbers of various quark flavors are conserved. But since quark states are not physically observed, one has to relate these flavor conservations into conservations of the experimentally observed charges of hadrons like baryon number $B$, electric charge $Q$, and strangeness $S$. The fluctuations and correlations of conserved charges depend significantly on the state of strongly interacting matter at high temperatures and densities $[17,56,57,163,164]$. Variations of these quantities with temperature and various chemical potentials are supposed to carry signatures of phase transition or crossover [16,65-67,127,143,165-169]. They are related to the susceptibilities of pressure via the fluctuation dissipation theorem [68]. The susceptibilities at various orders are easily obtained as a Taylor series expansion of pressure in terms of the corresponding chemical potentials. The diagonal Taylor coefficients $c_{n}^{X}(T)(X=B, Q, S)$ of $n$th order for the scaled pressure $P\left(T, \mu_{B}, \mu_{Q}, \mu_{S}\right) / T^{4}$ may be written in terms of the 

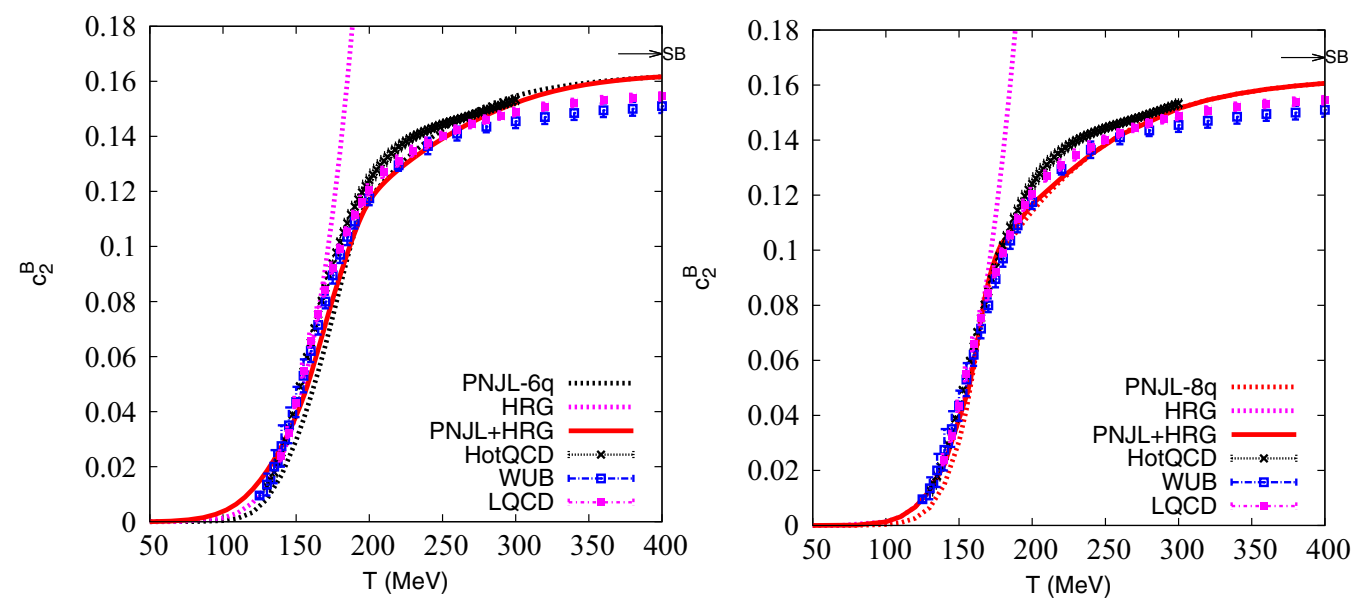

FIG. 5. Baryon number fluctuation as a function of temperature. The continuum extrapolated lattice data are from Ref. [104,105] (HotQCD), Ref. [106] (WUB), and Ref. [171] (LQCD).

fluctuations $\chi_{n}^{X}(T)$ of the corresponding order as

$$
c_{n}^{X}(T)=\frac{1}{n !} \frac{\partial^{n}\left(P / T^{4}\right)}{\partial\left(\frac{\mu_{X}}{T}\right)^{n}}=\frac{T^{n-4}}{n !} \chi_{n}^{X}(T),
$$

where the expansion is carried out around $\mu_{B}=\mu_{Q}=\mu_{S}=$ 0 . Similarly, the off-diagonal coefficients $c_{n, m}^{X, Y}(T)(X, Y=$ $B, Q, S ; X \neq Y)$ of the $(m+n)$ th order in the Taylor expansion of scaled pressure are related to the correlations between the conserved charges $\chi_{n, m}^{X, Y}(T)$ as

$$
c_{m, n}^{X, Y}=\frac{1}{m ! n !} \frac{\partial^{m+n}\left(P / T^{4}\right)}{\left[\partial\left(\frac{\mu_{X}}{T}\right)^{m}\right]\left[\partial\left(\frac{\mu_{Y}}{T}\right)^{n}\right]}=\frac{T^{m+n-4}}{m ! n !} \chi_{n, m}^{X, Y}(T) .
$$

At zero chemical potentials some of these fluctuations and correlations have been measured in the lattice QCD framework either in the continuum limit $[12,104,106,170,171]$ or for small lattice spacings close to the continuum limit [107]. We shall now discuss how much these quantities obtained in the PNJL-HRG model agree with the lattice QCD data. In the PNJL model the fluctuations and correlations are obtained by a suitable Taylor series fitting, as discussed in detail in [48]. On the other hand these quantities are obtained easily in the HRG model by taking the corresponding derivatives with respect to the chemical potentials. Once the fluctuations and correlations are obtained in both the models, they are combined into the PNJL-HRG model using the same switching function $S(T)$ identified in last section. They are given by

$$
\begin{gathered}
c_{n}^{X}=S(T) c_{n P}^{X}+[1-S(T)] c_{n H}^{X}, \\
\text { and } c_{m, n}^{X, Y}=S(T) c_{m, n P}^{X, Y}+[1-S(T)] c_{m, n}^{X, Y} H .
\end{gathered}
$$

As mentioned earlier we have assumed the switching function to be independent of the chemical potential in the close vicinity of zero chemical potentials. Let us now discuss some of these fluctuations and correlations obtained in the models and compare them to the lattice QCD data.

In Fig. 5 the variation of the baryon number susceptibility $c_{2}^{B}$ is shown as a function of temperature for various models and compared with lattice QCD data. Here we find $c_{2}^{B}$ obtained in the HRG model agrees with lattice QCD data up to about
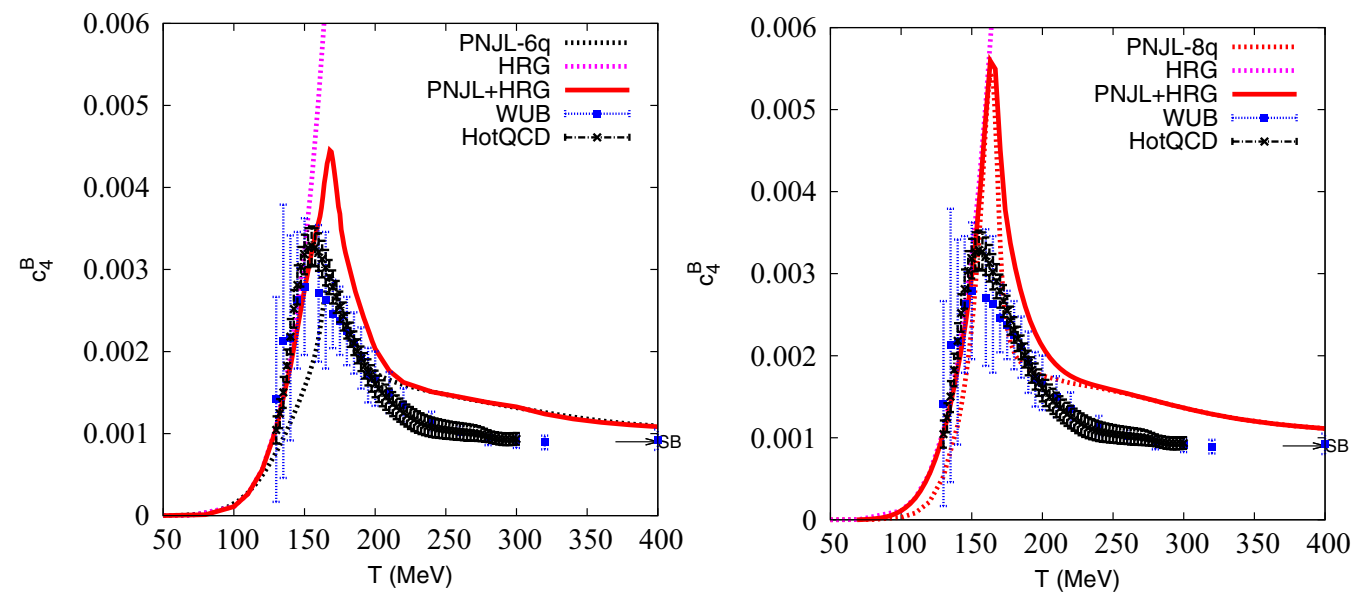

FIG. 6. Fourth-order baryon number susceptibility as function of temperature. The continuum extrapolated lattice data are from Ref. [104,105] (HotQCD) and Ref. [106] (WUB). 

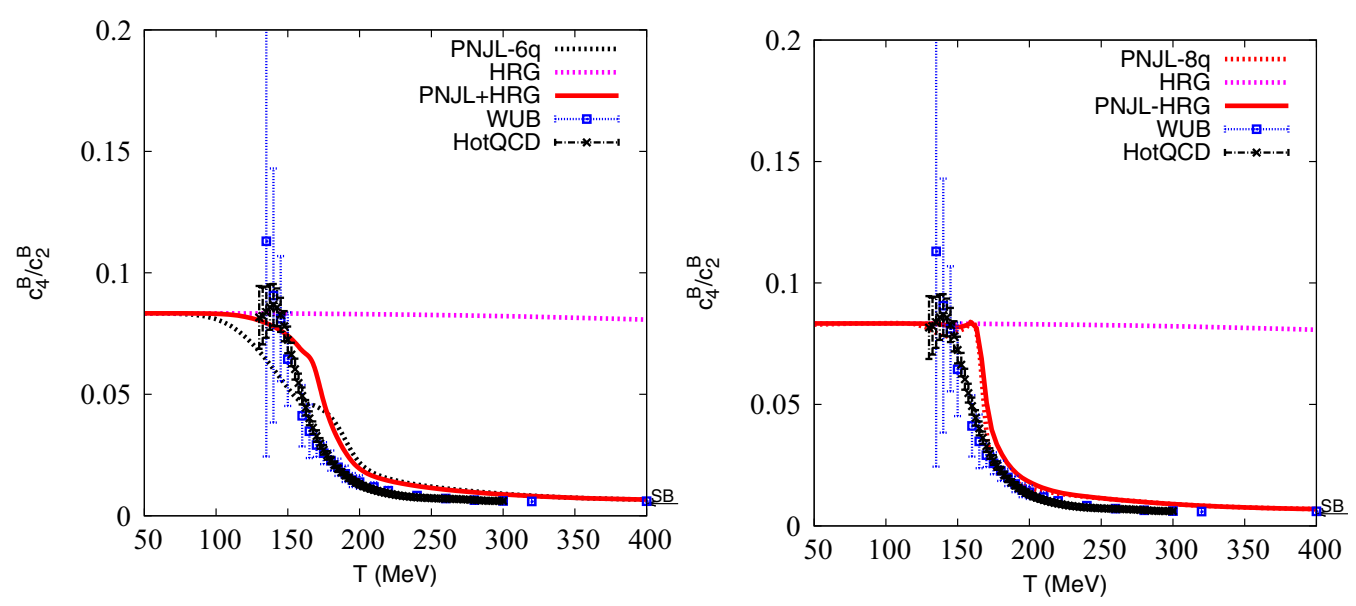

FIG. 7. Ratio of fourth-order to second-order baryon number susceptibility as function of temperature. The continuum extrapolated lattice data are from Ref. [104,105] (HotQCD) and Ref. [106] (WUB).

$T \sim 170 \mathrm{MeV}$. The PNJL model also agrees with lattice QCD quite well in this temperature range and therefore also the PNJL-HRG model. At higher temperatures the hybrid model is dominated by the PNJL model dynamics. There is a slight overestimation in the PNJL model which is also reflected in the PNJL-HRG model. Apart from that, the results in the six-quark and eight-quark versions of the PNJL models are numerically commensurate. The baryonic contribution of the PNJL model therefore seems to be sufficient in describing the strongly interacting matter even in the low temperature region described either by the HRG model or the lattice QCD data.

The temperature variation of the fourth-order baryon number susceptibility $c_{4}^{B}$ and the ratio $c_{4}^{B} / c_{2}^{B}$ related to kurtosis are shown in Figs. 6 and 7 respectively. In the region below $T_{c}$ the hybrid model results for $c_{4}^{B}$ agree quite well with the lattice continuum results. For $T>T_{c}$, however, the model overpredicts the $c_{4}^{B}$ commensurate with the corresponding contribution from the PNJL model. The resulting ratio $c_{4}^{B} / c_{2}^{B}$ agrees much better with the lattice data both below and above $T_{c}$.

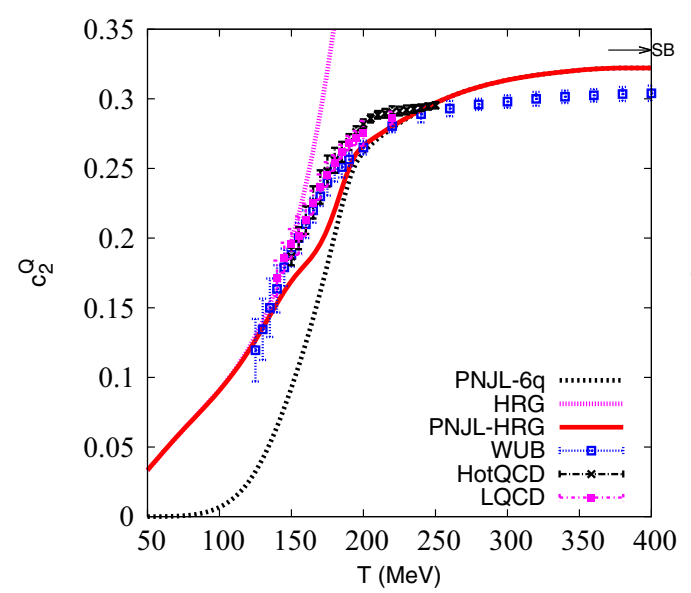

The variation of the electric charge susceptibility with temperature is shown in Fig. 8. There is a significant difference between the PNJL model and lattice QCD results for $c_{2}^{Q}$ below the crossover temperature $T_{c}$. The lattice data are much larger than the PNJL model results. Though the baryon fluctuations in the lattice data are well accounted for by the constituent quarks in the PNJL model, proper considerations of other hadronic degrees of freedom below $T_{c}$ are crucial to obtain the correct values of electric charge fluctuations. On the other hand lattice QCD data are reproduced by the HRG model very well for $T<150 \mathrm{MeV}$. This is expected, as the charge sector has dominant contributors from the light hadrons, which are practically absent in the PNJL model. Once the PNJL and HRG models are combined using the switching function, the PNJL-HRG model results again agree with the lattice QCD data very well.

The temperature variation of the strangeness susceptibility $c_{2}^{S}$ is shown in Fig. 9. The computations in the HRG model seem to agree with the lattice QCD data up to much higher temperatures $T \sim 190 \mathrm{MeV}$. This is surprising given that the crossover temperature, as well as the temperature around

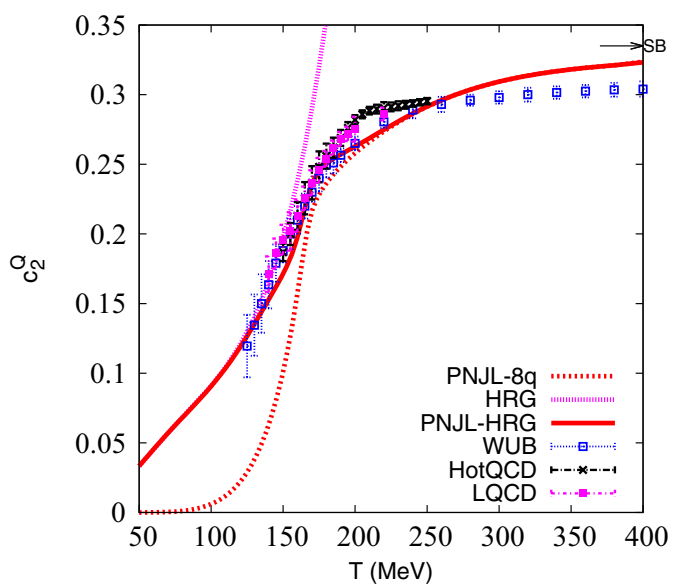

FIG. 8. Electric charge fluctuation as function of temperature. The continuum extrapolated lattice data are from Ref. [104] (HotQCD), Ref. [106] (WUB), and [171] (LQCD). 

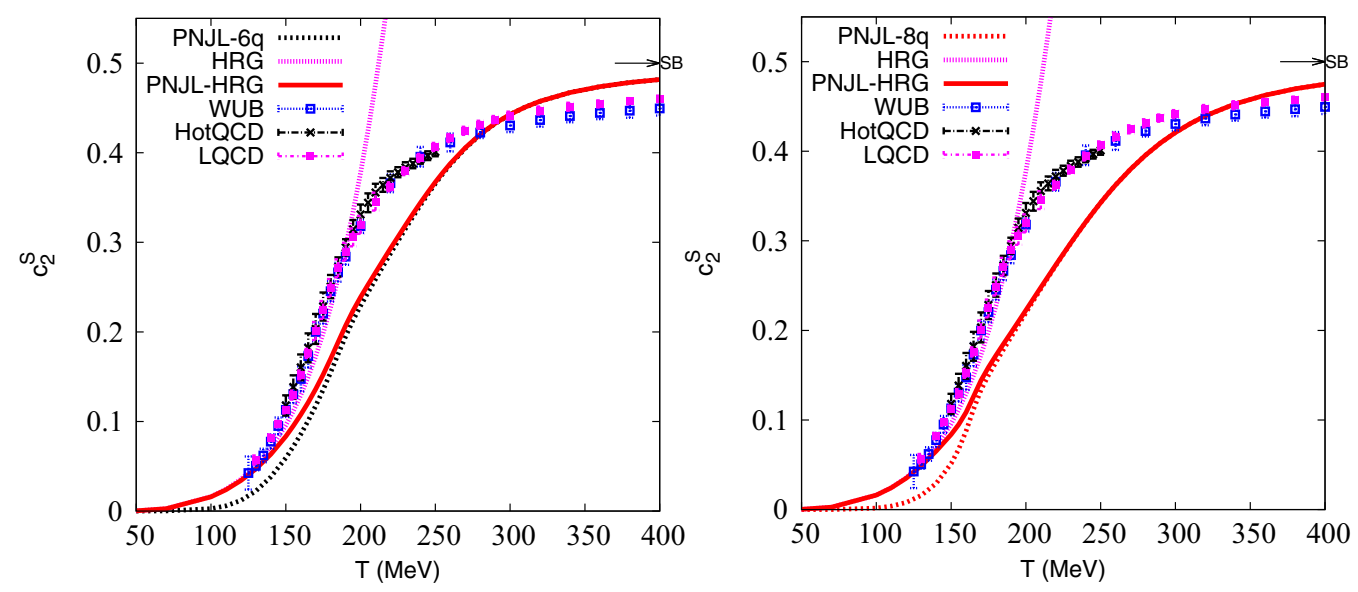

FIG. 9. Strangeness fluctuation as function of temperature. The continuum extrapolated lattice data are from Ref. [104] (HotQCD), Ref. [106] (WUB), and Ref. [171] (LQCD).

which almost all other quantities computed in the HRG model start deviating from lattice QCD data at around $T \sim 160$ $\mathrm{MeV}$. One would expect the $c_{2}^{S}$ in the HRG model to rise much faster and start deviating from lattice QCD data at much lower temperatures. One of the possible reasons for such a result may be that the HRG model is constructed from experimentally observed hadrons, whereas the lattice QCD formulation could have contributions from additional species of strange hadrons, which are also predicted by quark model calculations [108].

At the same time the quantitative results for $c_{2}^{S}$ obtained in PNJL model are significantly different from lattice QCD data up to $T \sim 250 \mathrm{MeV}$. The constituent masses of the strange quarks in the PNJL model have values above $500 \mathrm{MeV}$ for $T<150 \mathrm{MeV}$. This is consistent with the omega baryon mass. However, as the temperature rises, the constituent masses of strange quarks do not fall as fast as that of the light quarks. Therefore the strangeness fluctuations on their part do not rise as fast as that of the light quarks as observed in the lattice QCD data. This aspect of the PNJL model would need further scrutiny and will be discussed elsewhere. Note that the range of temperature where the PNJL model disagrees

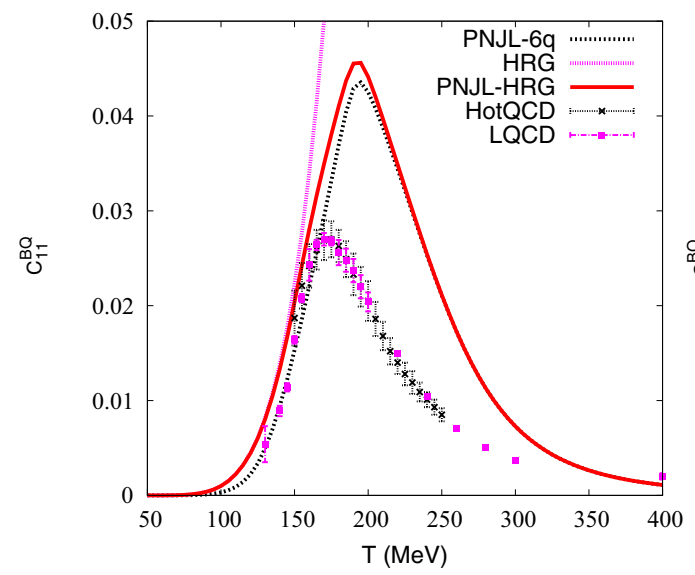

with lattice QCD data is above the range where the switching from HRG model to PNJL model takes place. Inclusion of the HRG model is therefore insufficient to address this issue. As a result the hybrid PNJL-HRG model does not agree well with the lattice QCD data. We thus encounter the first observable where the PNJL-HRG model could not satisfactorily describe the lattice QCD data in the full temperature range. It is apparent that this may be the case for other quantities that are strongly dependent on the strangeness content of the PNJL model.

We now discuss the leading order correlations between the conserved charges. The correlator $c_{11}^{B Q}$ between the baryon number and electric charge is shown in Fig. 10. In the hadronic phase the baryon and electric charges remain correlated as the baryons have positive electric charge and anti-baryons have negative electric charge. For small temperatures the correlations are small due to the relatively large masses. With increasing temperature however the correlation becomes nonzero. On the other hand for the $2+1$ flavor system there are three quarks with equal baryon number in the partonic phase, but electric charge of down and strange quarks are together opposite of that of the up quark. At large temperatures when

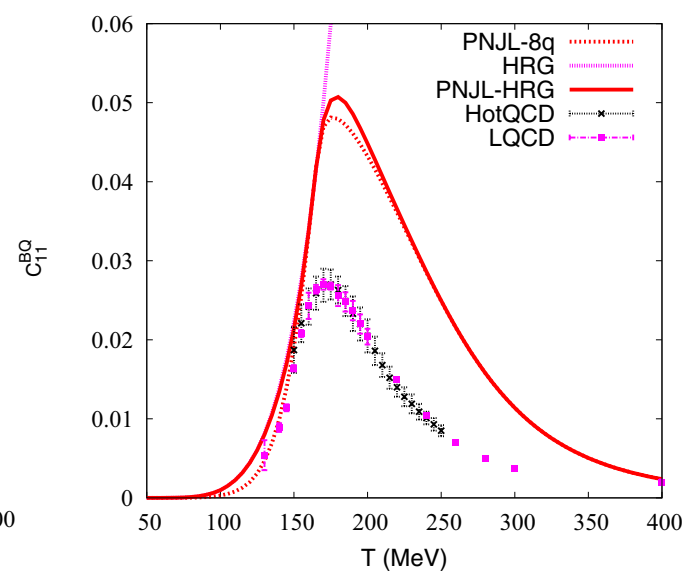

FIG. 10. Baryon-electric charge correlation as function of temperature. The continuum extrapolated lattice data are from Ref. [104] (HotQCD) and Ref. [171] (LQCD). 

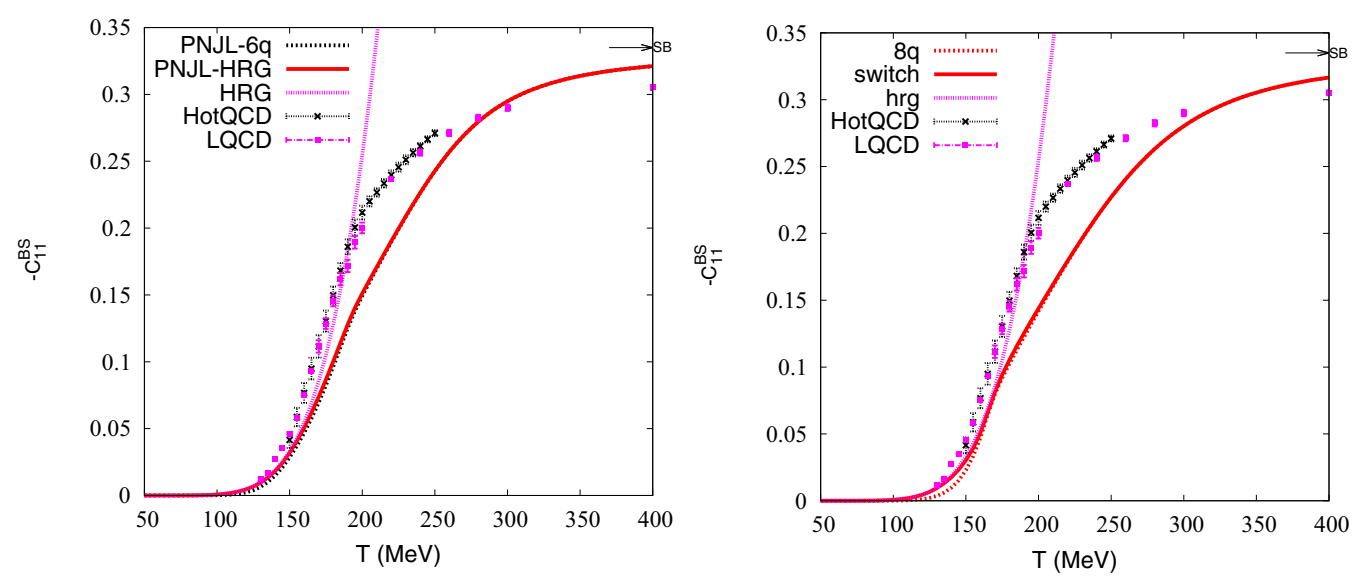

FIG. 11. Baryon-strangeness correlation as function of temperature. The continuum extrapolated lattice data are from Ref. [104] (HotQCD) and Ref. [171] (LQCD).

the quark masses are small with respect to the temperature, the baryon number to electric charge (BQ) correlation should again tend to zero. In the HRG model the BQ correlations keep on increasing as higher and higher mass states are getting excited with increase in temperature. On the other hand for lattice QCD data and PNJL model as well as any other model having a transition from hadronic to partonic phases, $c_{11}^{B Q}$ would show a hump around the crossover region. This is shown in Fig. 10. Here the BQ correlation in the PNJL model is larger than that obtained in the lattice QCD data for $T>150 \mathrm{MeV}$. It may again be anticipated that this is due to the slow decrease of the strange quark mass with temperature in the PNJL model. The number of strange quarks is therefore much smaller than that of the light quarks, and they cannot compensate the electric charge of the up quarks sufficiently.

The baryon number to strangeness (BS) correlation $c_{11}^{B S}$ is shown in Fig. 11 and the electric charge to strangeness (QS) correlation $c_{11}^{Q S}$ is shown in Fig. 12. For the intermediate temperatures the BS correlation is a little less than the QS correlation due to the contributions from lighter strange mesons in the latter. In the HRG model, these two correlations again keep on increasing indefinitely as higher and higher hadronic states are getting excited. The behavior in the lattice QCD data as well as the PNJL model is as it should be in a theory that has partons in the high temperature phase. This can be understood by noting that at low temperatures the correlators are small due to the large hadronic masses. They will increase with temperature, and in the partonic phase the correlation saturates as there is only one species of quarks containing both strangeness and baryon number or strangeness and electric charge.

As in the case of $c_{2}^{S}$, the BS and QS correlators in the PNJL-HRG model underestimate the lattice QCD data in the intermediate range of temperatures possibly due to the slowly decreasing strange quark mass in the PNJL model. Therefore it seems that this deviation would pervade all other observables related to strangeness. A reparametrization of the NJL part of the model may be able to address this issue and will be discussed elsewhere.

\section{CONCLUSION}

In this study we discussed a scheme to address the inadequacy of the PNJL model in describing the hadronic state of matter as pointed out in our earlier work in [109].
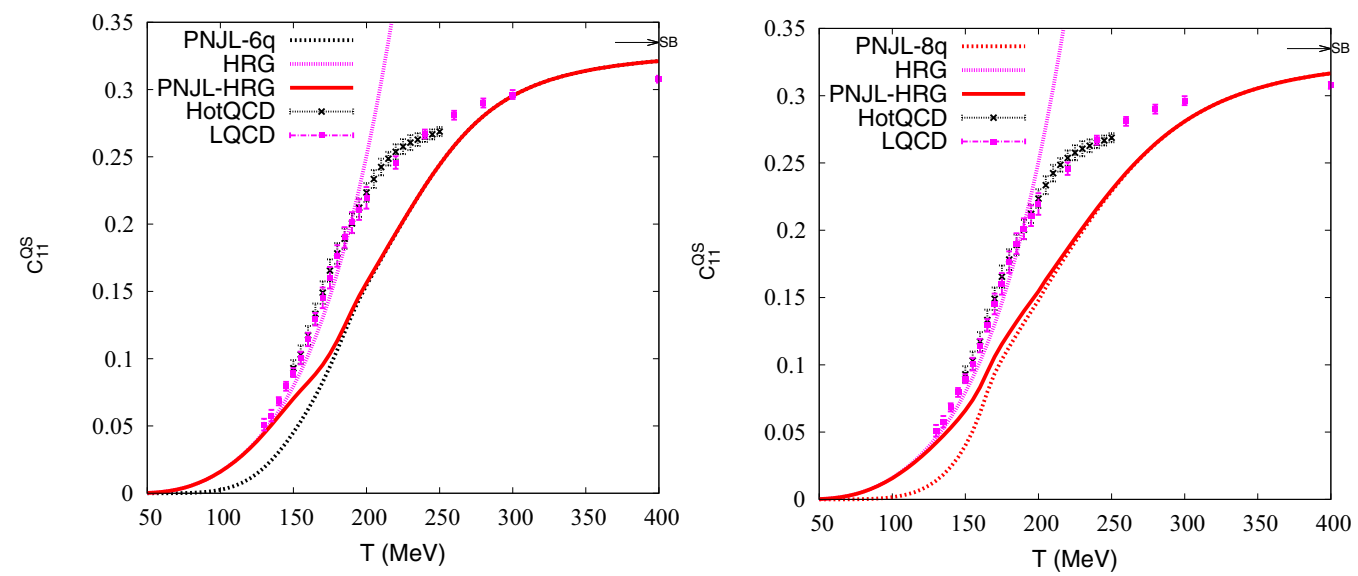

FIG. 12. Electric charge-strangeness correlation as function of temperature. The continuum extrapolated lattice data are from Ref. [104] (HotQCD) and Ref. [171] (LQCD). 
A straightforward approach would be to add the hadronic contribution from the HRG model. However a simple addition of HRG model to PNJL model would have led to overcounting the degrees of freedom. An interpolating function was therefore used in line with some earlier studies [146-150], to smoothly switch between the hadronic and partonic matter. In general this function would be dependent on temperature and chemical potentials. Since we have discussed various thermodynamic observables vis-a-vis lattice QCD data at zero chemical potentials, we have considered only a temperature dependent switching function.

In the earlier studies with the switching function, its temperature and chemical potential derivatives were considered for computing observables from thermodynamic relations. However we considered the switching function to be independent of the observable chosen. Otherwise the various derivatives of the switching function may be obtained by fitting with as many number of observables from lattice QCD see e.g. [150]. This would increase the number of free parameters in the model, thereby reducing its predictive power. Instead we emphasized on the better agreement of the PNJL model and lattice QCD data above the crossover region. Therefore a considerable part of the equation of state in continuum lattice QCD data, both below and above the crossover temperature, was well described by HRG and PNJL models respectively. Hence we only needed to interpolate in the crossover region using the switching function. Thereafter all other observables were obtained from thermodynamic relations acting on the individual pressure of the two models weighed with the switching function. For completeness however we have made a comparative study of observables related to the equation of state by both including and excluding these derivatives. All the thermodynamic quantities computed in the PNJL-HRG model reproduced the lattice QCD data quite well.

Once the switching function was fixed, we computed the predicted behavior of various fluctuations and correlations of conserved charges. We found that the fluctuations of baryon number and electric charge computed in the PNJL-HRG model is in good quantitative agreement with the lattice QCD data. However the strangeness fluctuations in the PNJL model was somewhat different from the lattice QCD data around the crossover region. As a result the PNJL-HRG model did not reproduce lattice QCD data satisfactorily. We emphasize that this disagreement is not due to the switching function but rather the inadequacy in the PNJL model. We argued that since in the PNJL model the constituent mass of the strange quarks does not decrease fast enough with the rising temperature, there is a departure of fluctuations of strangeness from the lattice QCD data. It naturally followed that none of the leading order correlators in the PNJL-HRG model could reproduce lattice QCD data as well.

Finally we would like to conclude that the scheme of introducing the HRG model to the PNJL model using the switching function seems to have worked well. Discrepancies, if any, are only due to difference between the PNJL model and lattice QCD data in the relevant temperature ranges. Apart from the considerations of the PNJL model it may also be important to incorporate interactions in the HRG model itself. For example we have not included the effects of the hadron mass modifications. This is the usual practice for the HRG model, where the PDG masses are considered independent of medium effects. As another example, in an interesting work [172] it was pointed out that the contribution of the resonance $f 0$ (500) to isospin-averaged observables is to a great extent canceled by the repulsion from the isotensor-scalar channel. These kind of effects in various other channels may lead to significant changes in the thermodynamic contributions in the hadronic sector. Further, for treading into the nonzero chemical potential regions we may have to consider a chemical potential dependence of the switching function. We hope to address these issues elsewhere.

\section{ACKNOWLEDGMENTS}

The authors would like to thank the Council for Scientific and Industrial Research (CSIR), Department of Science and Technology (DST), Department of Atomic Energy (DAE), and Board of Research for Nuclear Sciences (BRNS) for financial support. K.S. acknowledges the financial support from DST-SERB under NPDF Project No. PDF/2017/002399. We would like to thank Swagato Mukherjee for providing us with various lattice QCD data and many useful discussions.
[1] H.-T. Ding, F. Karsch, and S. Mukherjee, in Quark Gluon Plasma 5, edited by X. N. Wang (World Scientific, Singapore, 2016).

[2] G. Boyd, J. Engels, F. Karsch, E. Laermann, C. legeland, M. Lugermeier, and B. Peterson, Nucl. Phys. B 469, 419 (1996).

[3] J. Engels, O. Kaczmarek, F. Karsch, and E. Laermann, Nucl. Phys. B 558, 307 (1999).

[4] Z. Fodor and S. D. Katz, Phys. Lett. B 534, 87 (2002).

[5] C. R. Allton, S. Ejiri, S. J. Hands, O. Kaczmarek, F. Karsch, E. Laermann Ch. Schmidt, and L. Scorzato, Phys. Rev. D 66, 074507 (2002).

[6] Z. Fodor, S. D. Katz, and K. K. Szabo, Phys. Lett. B 568, 73 (2003).

[7] C. R. Allton, S. Ejiri, S. J. Hands, O. Kaczmarek, F. Karsch, E. Laermann, and Ch. Schmidt, Phys. Rev. D 68, 014507 (2003).
[8] P. de Forcrand and O. Philipsen, Nucl. Phys. B 673, 170 (2003).

[9] Y. Aoki, Z. Fodor, S. D. Katz, and K. K. Szabo, Phys. Lett. B 643, 46 (2006).

[10] Y. Aoki, G. Endrodi, Z. Fodor, S. D. Katz, and K. K. Szabo, Nature (London) 443, 675 (2006).

[11] Y. Aoki, S. Borsanyi, S. Durr, Z. Fodor, S. D. Katz, S. Krieg, and K. K. Szabo, J. High Energy Phys. 06 (2009) 088.

[12] S. Borsanyi, Z. Fodor, S. D. Katz, S. Krieg, C. Ratti, and K. K. Szabo, Phys. Rev. Lett. 111, 062005 (2013).

[13] A. Bazavov et al. (HotQCD Collaboration), Phys. Rev. D 85, 054503 (2012).

[14] A. Bazavov et al. (HotQCD Collaboration), Phys. Rev. D 90, 094503 (2014). 
[15] S. Borsanyi, Z. Fodor, C. Hoelbling, S. D. Katz, S. Krieg, and K. K. Szabo, Phys. Lett. B 730, 99 (2014).

[16] Y. Hatta and M. A. Stephanov, Phys. Rev. Lett. 91, 102003 (2003); 91, 129901(E) (2003).

[17] S. Ejiri, F. Karsch, and K. Redlich, Phys. Lett. B 633, 275 (2006).

[18] M. A. Stephanov, Phys. Rev. Lett. 102, 032301 (2009).

[19] M. Asakawa and K. Yazaki, Nucl. Phys. A 504, 668 (1989).

[20] S. Ejiri, Phys. Rev. D 78, 074507 (2008).

[21] E. S. Bowman and J. I. Kapusta, Phys. Rev. C 79, 015202 (2009).

[22] M. A. Halasz, A. D. Jackson, R. E. Shrock, M. A. Stephanov, and J. J. M. Verbaarschot, Phys. Rev. D 58, 096007 (1998).

[23] Z. Fodor and S. D. Katz, J. High Energy Phys. 04 (2004) 050.

[24] R. V. Gavai and S. Gupta, Phys. Rev. D 71, 114014 (2005).

[25] M. A. Stephanov, Int. J. Mod. Phys. A 20, 4387 (2005).

[26] S. Gottlieb, W. Liu, D. Toussaint, R. L. Renken, and R. L. Sugar, Phys. Rev. Lett. 59, 2247 (1987); S. Choe et al. (QCDTARO Collaboration), Phys. Rev. D 65, 054501 (2002).

[27] R. V. Gavai, S. Gupta, and P. Majumdar, Phys. Rev. D 65, 054506 (2002).

[28] M. D’Elia and M.-P. Lombardo, Phys. Rev. D 67, 014505 (2003).

[29] R. V. Gavai and S. Gupta, Phys. Rev. D 68, 034506 (2003).

[30] S. Gupta and R. Ray, Phys. Rev. D 70, 114015 (2004); R. Gavai, S. Gupta, and R. Ray, Prog. Theor. Phys. Suppl. 153, 270 (2004); R. V. Gavai and S. Gupta, Phys. Rev. D 72, 054006 (2005).

[31] C. R. Allton, M. Döring, S. Ejiri, S. J. Hands, O. Kaczmarek, F. Karsch, E. Laermann, and K. Redlich, Phys. Rev. D 71, 054508 (2005).

[32] C. Bernard, T. Burch, C. DeTar, J. Osborn, S. Gottlieb, E. B. Gregory, D. Toussaint, U. M. Heller, and R. Sugar (MILC Collaboration), Phys. Rev. D 71, 034504 (2005).

[33] C. Bernard, C. E. DeTar, L. Levkova, S. Gottlieb, U. M. Heller, J. E. Hetrick, R. Sugar, and D. Toussaint, Phys. Rev. D 77, 014503 (2008).

[34] M. Cheng et al., Phys. Rev. D 77, 014511 (2008).

[35] O. Kaczmarek, F. Karsch, E. Laermann, C. Miao, S. Mukherjee, P. Petreczky, C. Schmidt, W. Soeldner, and W. Unger, Phys. Rev. D 83, 014504 (2011).

[36] G. Endrodi, Z. Fodor, S. D. Katz, and K. K. Szabo, J. High Energy Phys. 04 (2011) 001.

[37] S. Borsanyi, G. Endrodi, Z. Fodor, S. D. Katz, S. Krieg, C. Ratti, and K. K. Szabo, J. High Energy Phys. 08 (2012) 053.

[38] Y. Nambu and G. Jona-Lasinio, Phys. Rev. 122, 345 (1961); 124, 246 (1961).

[39] T. Kunihiro and T. Hatsuda, Phys. Lett. B 206, 385 (1988).

[40] U. Vogl and W. Weise, Prog. Part. Nucl. Phys. 27, 195 (1991).

[41] S. P. Klevansky, Rev. Mod. Phys. 64, 649 (1992).

[42] T. Hatsuda and T. Kunihiro, Phys. Rep. 247, 221 (1994).

[43] M. Buballa, Phys. Rep. 407, 205 (2005).

[44] A. Barducci, R. Casalbuoni, G. Pettini, and L. Ravagli, Phys. Rev. D 72, 056002 (2005).

[45] P. N. Meisinger and M. C. Ogilvie, Phys. Lett. B 379, 163 (1996); Nucl. Phys. B Proc. Suppl. 47, 519 (1996).

[46] K. Fukushima, Phys. Lett. B 591, 277 (2004).

[47] C. Ratti, M. A. Thaler, and W. Weise, Phys. Rev. D 73, 014019 (2006).

[48] S. K. Ghosh, T. K. Mukherjee, M. G. Mustafa, and R. Ray, Phys. Rev. D 73, 114007 (2006).
[49] S. Mukherjee, M. G. Mustafa, and R. Ray, Phys. Rev. D 75, 094015 (2007).

[50] C. Ratti, S. Robner, and W. Weise, Phys. Lett. B 649, 57 (2007).

[51] S. K. Ghosh, T. K. Mukherjee, M. G. Mustafa, and R. Ray, Phys. Rev. D 77, 094024 (2008).

[52] P. Deb, A. Bhattacharyya, and S. Datta, and S. K. Ghosh, Phys. Rev. C 79, 055208 (2009).

[53] W.-J. Fu, Y.-X. Liu, and Y.-L. Wu, Phys. Rev. D 81, 014028 (2010).

[54] W.-j. Fu and Y.-1. Wu, Phys. Rev. D 82, 074013 (2010).

[55] A. Bhattacharyya, P. Deb, S. K. Ghosh, and R. Ray, Phys. Rev. D 82, 014021 (2010).

[56] A. Bhattacharyya, P. Deb, A. Lahiri, and R. Ray, Phys. Rev. D 82, 114028 (2010).

[57] A. Bhattacharyya, P. Deb, A. Lahiri, and R. Ray, Phys. Rev. D 83, 014011 (2011).

[58] A. A. Osipov, B. Hiller, and J. da Providěncia, Phys. Lett. B 634, 48 (2006).

[59] A. A. Osipov, B. Hiller, A. H. Blin, and J. da Providência, Ann. Phys. (NY) 322, 2021 (2007).

[60] A. A. Osipov, B. Hiller, J. Moreira, A. H. Blin, and J. da Providência, Phys. Lett. B 646, 91 (2007).

[61] B. Hiller, J. Moreira, A. A. Osipov, and A. H. Blin, Phys. Rev. D 81, 116005 (2010).

[62] A. Bhattacharyya, S. K. Ghosh, S. Majumder, and R. Ray, Phys. Rev. D 86, 096006 (2012).

[63] C. A. Islam, R. Abir, M. G. Mustafa, R. Ray, and S. K. Ghosh, J. Phys. G 41, 025001 (2014).

[64] A. Bhattacharyya, S. K. Ghosh, A. Lahiri, S. Majumder, S. Raha, and R. Ray, Phys. Rev. C 89, 064905 (2014).

[65] A. Bhattacharyya, P. Deb, S. K. Ghosh, R. Ray, and S. Sur, Phys. Rev. D 87, 054009 (2013).

[66] A. Bhattacharyya, R. Ray, and S. Sur, Phys. Rev. D 91, 051501(R) (2015).

[67] A. Bhattacharyya, S. Das, S. K. Ghosh, S. Raha, R. Ray, K. Saha, and S. Upadhaya, arXiv:1212.6010.

[68] S. K. Ghosh, A. Lahiri, S. Majumder, M. G. Mustafa, S. Raha, and R. Ray, Phys. Rev. D 90, 054030 (2014).

[69] C. Sasaki and K. Redlich, Nucl. Phys. A 832, 62-75 (2010).

[70] S. Ghosh, A. Lahiri, S. Majumder, R. Ray, and S. K. Ghosh, Phys. Rev. C 88, 068201 (2013).

[71] R. Marty, E. Bratkovskaya, W. Cassing, J. Aichelin, and H. Berrehrah, Phys. Rev. C 88, 045204 (2013).

[72] R. Lang and W. Weise, Eur. Phys. J. A 50, 63 (2014).

[73] X. Shi-Song, G. Pan-Pan, Z. Le, and H. De-Fu, Chinese Phys. C 38, 054101 (2014).

[74] S. K. Ghosh, S. Raha, R. Ray, K. Saha, and S. Upadhaya, Phys. Rev. D 91, 054005 (2015).

[75] K. Saha, S. Upadhaya, and S. Ghosh, Mod. Phys. Lett. A 32, 1750018 (2017).

[76] R. Lang, N. Kaiser, and W. Weise, Eur. Phys. J. A 51, 127 (2015).

[77] S. Ghosh, S. K. Das, V. Greco, S. Sarkar, and Jan-e Alam, Phys. Rev. D 90, 054018 (2014).

[78] S. Ghosh, G. Krein, and S. Sarkar, Phys. Rev. C 89, 045201 (2014).

[79] G. P. Kadam and H. Mishra, Phys. Rev. C 92, 035203 (2015).

[80] S. Ghosh, S. Chatterjee, and B. Mohanty, Phys. Rev. C 94, 045208 (2016). 
[81] S. Rößner, C. Ratti, and W. Weise, Phys. Rev. D 75, 034007 (2007).

[82] C. Sasaki, B. Friman, and K. Redlich, Phys. Rev. D 75, 074013 (2007).

[83] K. Fukushima, Phys. Rev. D 77, 114028 (2008); 78, 039902(E) (2008).

[84] T. Kahara and K. Tuominen, Phys. Rev. D 78, 034015 (2008).

[85] W.-j. Fu, Z. Zhang, and Y.-x. Liu, Phys. Rev. D 77, 014006 (2008).

[86] P. Costa, M. C. Ruivo, and C. A. de Sousa, Phys. Rev. D 77, 096001 (2008).

[87] K. Kashiwa, H. Kouno, M. Matsuzaki, and M. Yahiro, Phys. Lett. B 662, 26 (2008).

[88] M. Buballa, A. G. Grunfeld, A. E. Radzhabov, and D. Scheffler, Prog. Part. Nucl. Phys. 62, 365 (2009).

[89] P. Costa, H. Hansen, M. C. Ruivo, and C. A. de Sousa, Phys. Rev. D 81, 016007 (2010).

[90] O. Lourenco, M. Dutra, A. Delfino, and M. Malheiro, Phys. Rev. D 84, 125034 (2011).

[91] T. Inagaki, D. Kimura, H. Kohyama, and A. Kvinikhidze, Phys. Rev. D 86, 116013 (2012).

[92] A. V. Friesen, Y. U. L. Kalinovsky, and V. D. Toneev, Int. J. Mod. Phys. A 27, 1250013 (2012).

[93] Y. Sakai, K. Kashiwa, H. Kouno, and M. Yahiro, Phys. Rev. D 77, 051901(R) (2008); 78, 036001(E) (2008).

[94] Y. Sakai, H. Kouno, and M. Yahiro, J. Phys. G 37, 105007 (2010).

[95] K. Morita, V. Skokov, B. Friman, and K. Redlich, Phys. Rev. D 84, 076009 (2011).

[96] E. Megías, E. R. Arriola, and L. L. Salcedo, J. High Energy Phys. 01 (2006) 073.

[97] E. Megías, E. R. Arriola, and L. L. Salcedo, Phys. Rev. Lett. 109, 151601 (2012).

[98] E. Megías, E. R. Arriola, and L. L. Salcedo, Phys. Rev. D 89, 076006 (2014).

[99] E. Megías, E. R. Arriola, and L. L. Salcedo, Phys. Rev. D 74, 065005 (2006).

[100] E. Megías, E. R. Arriola, and L. L. Salcedo, Phys. Rev. D 74, 114014 (2006).

[101] H.-M. Tsai and B. Muller, J. Phys. G 36, 075101 (2009).

[102] J. Braun, L. M. Haas, F. Marhauser, and J. M. Pawlowski, Phys. Rev. Lett. 106, 022002 (2011); L. M. Haas, R. Stiele, J. Braun, J. M. Pawlowski, and J. Schaffner-Bielich, Phys. Rev. D 87, 076004 (2013).

[103] J. M. Torres-Rincon and J. Aichelin, arXiv:1601.01706; Phys. Rev. C 96, 045205 (2017).

[104] A. Bazavov et al. (HotQCD Collaboration), Phys. Rev. D 86, 034509 (2012); A. Bazavov, H.-T. Ding, P. Hegde, F. Karsch, C. Miao, S. Mukherjee, P. Petreczky, C. Schmidt, and A. Velytsky, ibid. 88, 094021 (2013); H.-T. Ding, Swagato Mukherjee, H. Ohno, and P. Petreczky, and H.-P. Schadler, ibid. 92, 074043 (2015); A. Bazavov et al., ibid. 95, 054504 (2017).

[105] A. Bazavov et al. (HotQCD Collaboration), Phys. Rev. D 96, 074510 (2017).

[106] S. Borsanyi, Z. Fodor, S. D. Katz, S. Krieg, C. Ratti, and K. Szabo, J. High Energy Phys. 01 (2012) 138.

[107] A. Bazavov et al., Phys. Rev. Lett. 109, 192302 (2012); C. Schmidt (for the BNL-Bielefeld Collaboration),
PoS(Confinement X2012)187 (2012); A. Bazavov et al., Phys. Rev. Lett. 111, 082301 (2013).

[108] A. Bazavov et al., Phys. Rev. Lett. 113, 072001 (2014).

[109] A. Bhattacharyya, S. K. Ghosh, S. Maity, S. Raha, R. Ray, K. Saha, and S. Upadhaya, Phys. Rev. D 95, 054005 (2017).

[110] P. Braun-Munzinger, K. Redlich, and J. Stachel, in Quark Gluon Plasma 3, edited by R. C. Hwa and X. N. Wang (World Scientific, Singapore, 2004).

[111] P. Braun-Munzinger, J. Stachel, J. P. Wessels, and N. Xu, Phys. Lett. B 344, 43 (1995).

[112] J. Cleymans, D. Elliott, H. Satz, and R. L. Thews, Z. Phys. C 74, 319 (1997).

[113] P. Braun-Munzinger, I. Heppe, and J. Stachel, Phys. Lett. B 465, 15 (1999).

[114] J. Cleymans and K. Redlich, Phys. Rev. C 60, 054908 (1999).

[115] P. Braun-Munzinger, D. Magestro, K. Redlich, and J. Stachel, Phys. Lett. B 518, 41 (2001).

[116] N. Xu and M. Kaneta, Nucl. Phys. A 698, 306 (2002).

[117] F. Becattini, J. Manninen, and M. Gazdzicki, Phys. Rev. C 73, 044905 (2006).

[118] A. Andronic, P. Braun-Munzinger, and J. Stachel, Nucl. Phys. A 772, 167 (2006).

[119] J. Cleymans, H. Oeschler, K. Redlich, and S. Wheaton, Phys. Rev. C 73, 034905 (2006).

[120] A. Andronic, P. Braun-Munzinger, and J. Stachel, Phys. Lett. B 673, 142 (2009).

[121] A. Andronic, P. Braun-Munzinger, and J. Stachel, Nucl. Phys. A 834, 237C (2010).

[122] F. Karsch and K. Redlich, Phys. Lett. B 695, 136 (2011).

[123] S. Chatterjee, S. Das, L. Kumar, D. Mishra, B. Mohanty, R. Sahoo, and N. Sharma, Adv. High Energy Phys. 2015, 349013 (2015).

[124] F. Karsch, K. Redlich, and A. Tawfik, Phys. Lett. B 571, 67 (2003).

[125] A. Tawfik, Phys. Rev. D 71, 054502 (2005).

[126] A. Andronic, P. Braun-Munzinger, J. Stachel, and M. Winn, Phys. Lett. B 718, 80 (2012).

[127] A. Bhattacharyya, S. Das, S. K. Ghosh, R. Ray, and S. Samanta, Phys. Rev. C 90, 034909 (2014).

[128] R. Dashen, S.-K. Ma, and H. J. Bernstein, Phys. Rev. 187, 345 (1969); R. Dashen and S.-K. Ma, Phys. Rev. A 4, 700 (1971).

[129] R. Hagedorn and J. Rafelski, Phys. Lett. B 97, 136 (1980).

[130] D. H. Rischke, M. I. Gorenstein, H. Stöcker, and W. Greiner, Z. Phys. C 51, 485 (1991).

[131] J. Cleymans, M. I. Gorenstein, J. Stalnacke, and E. Suhonen, Phys. Scr. 48, 277 (1993).

[132] C. P. Singh, B. K. Patra, and K. K. Singh, Phys. Lett. B 387, 680 (1996).

[133] G. D. Yen, M. I. Gorenstein, W. Greiner, and S. N. Yang, Phys. Rev. C 56, 2210 (1997).

[134] M. I. Gorenstein, M. Hauer, and O. N. Moroz, Phys. Rev. C 77, 024911 (2008).

[135] J. Fu, Phys. Rev. C 85, 064905 (2012).

[136] V. V. Begun, M. Gaździcki, and M. I. Gorenstein, Phys. Rev. C 88, 024902 (2013).

[137] J. Fu, Phys. Lett. B 722, 144 (2013).

[138] A. Tawfik, Phys. Rev. C 88, 035203 (2013).

[139] Y. Hama, T. Kodama, and O. Socolowski, Braz. J. Phys. 35, 24 (2005). 
[140] K. Werner, Iu. Karpenko, T. Pierog, M. Bleicher, and K. Mikhailov, Phys. Rev. C 82, 044904 (2010).

[141] A. V. Merdeev, L. M. Satarov, and I. N. Mishustin, Phys. Rev. C 84, 014907 (2011).

[142] P. Garg, D. K. Mishra, P. K. Netrakanti, B. Mohanty, A. K. Mohanty, B. K. Singh, and N. Xu, Phys. Lett. B 726, 691 (2013).

[143] A. Bhattacharyya, R. Ray, S. Samanta, and S. Sur, Phys. Rev. C 91, 041901(R) (2015).

[144] A. Bhattacharyya, S. K. Ghosh, R. Ray, and S. Samanta, Europhys. Lett. 115, 62003 (2016).

[145] R. P. Adak, S. Das, S. K. Ghosh, R. Ray, and S. Samanta, Phys. Rev. C 96, 014902 (2017).

[146] M. Albright, J. Kapusta, and C. Young, Phys. Rev. C 90, 024915 (2014).

[147] M. Albright, J. Kapusta, and C. Young, Phys. Rev. C 92, 044904 (2015).

[148] J. Kapusta, M. Albright, and C. Young, Eur. Phys. J. A 52, 250 (2016).

[149] A. Miyahara, Y. Torigoe, H. Kouno, and M. Yahiro, Phys. Rev. D 94, 016003 (2016).

[150] A. Miyahara, M. Ishii, H. Kouno, and M. Yahiro, Int. J. Mod. Phys. A 321750205 (2017).

[151] V. I. Yukalov and E. P. Yukalova, Physica A 243, 382 (1997); Phys. Part. Nucl. 28, 37 (1997); PoS(Baldin ISHEPP XXI)046 (2012).

[152] M. Ciminale, R. Gatto, N. D. Ippolito, G. Nardulli, and M. Ruggieri, Phys. Rev. D 77, 054023 (2008).

[153] G.-y. Shao, Z.-d. Tang, M. Di Toro, M. Colonna, X.-y. Gao, and N. Gao, Phys. Rev. D 94, 014008 (2016).

[154] G.-y. Shao, Z.-d. Tang, M. Di Toro, M. Colonna, X.-y. Gao, N. Gao, and Y. L. Zhao, Phys. Rev. D 92, 114027 (2015).

[155] C. A. Islam, S. Majumder, N. Haque, and M. G. Mustafa, J. High Energy Phys. 02 (2015) 011.
[156] S. Ghosh, T. C. Peixoto, V. Roy, F. E. Serna, and G. Krein, Phys. Rev. C 93, 045205 (2016).

[157] J. Moreira, B. Hiller, A. A. Osipov, and A. H. Blin, Int. J. Mod. Phys. A 27, 1250060 (2012).

[158] G. A. Contrera, A. G. Grunfeld, and D. B. Blaschke, Phys. Part. Nucl. Lett. 11, 342 (2014).

[159] X.-y. Xin, S.-x. Qin, and Y. X. Liu, Phys. Rev. D 89, 094012 (2014).

[160] A. Bazavov, N. Brambilla, H.-T. Ding, P. Petreczky, H.-P. Schadler, A. Vairo, and J. H. Weber (TUMQCD Collaboration), Phys. Rev. D 93, 114502 (2016).

[161] K. A. Olive et al. (Particle Data Group), Chin. Phys. C 38, 090001 (2014).

[162] C. M. Hung and E. V. Shuryak, Phys. Rev. Lett. 75, 4003 (1995).

[163] V. Koch, in Relativistic Heavy Ion Physics, edited by R. Stock, Landolt-Börnstein New Series I, Vol. 23 (Springer, Heidelberg, 2010), pp. 626-652.

[164] B. Abelev et al., Phys. Rev. Lett. 110, 152301 (2013).

[165] M. A. Stephanov, K. Rajagopal, and E. V. Shuryak, Phys. Rev. Lett. 81, 4816 (1998).

[166] S. Jeon and V. Koch, Phys. Rev. Lett. 83, 5435 (1999).

[167] S. Jeon and V. Koch, Phys. Rev. Lett. 85, 2076 (2000).

[168] M. Asakawa, U. Heinz, and B. Muller, Phys. Rev. Lett. 85, 2072 (2000).

[169] A. Bhattacharyya, S. K. Ghosh, R. Ray, K. Saha, and S. Upadhaya, Europhys. Lett. 116, 52001 (2016).

[170] R. Bellwied, S. Borsanyi, Z. Fodor, S. D. Katz, and C. Ratti, Phys. Rev. Lett 111, 202302 (2013).

[171] R. Bellwied, S. Borsanyi, Z. Fodor, S. D. Katz, A. Pasztor, C. Ratti, and K. K. Szabo, Phys. Rev. D 92, 114505 (2015).

[172] W. Broniowski, F. Giacosa, and V. Begun, Phys. Rev. C 92, 034905 (2015). 